\title{
Distributed fibre optic sensing of strains on buried full-scale PVC pipelines crossing a normal fault
}

\author{
P. NI*, I. D. MOORE* and W. A. TAKE*
}

\begin{abstract}
Seismic faulting is extremely detrimental to buried pipelines owing to bending moments and axial forces generated by the soil-pipe interaction. Pipe responses to ground rupture are usually evaluated by beam-on-spring analysis. However, the empirical data used to define the spring response were obtained for steel pipes with high flexural stiffness and the effectiveness of the resulting spring stiffness values for flexible pipes is questionable. This paper presents full-scale tests undertaken using a new split-box apparatus which permits the testing of pipes in dry sand subject to a normal fault with dip angle of $90^{\circ}$. Four polyvinyl chloride (PVC) pipes (various diameters), one instrumented with strain gauges and three instrumented with fibre optic sensors, were tested to provide experimental evidence for flexible pipes. The research includes confirmation of the effectiveness of fibre optic strain measurement, which has the advantage of providing much more data than the limited discrete values obtained from conventional strain gauges. However, the fibre optic strain sensing technique is limited to strain values below $1 \%$. The prototype-scale testing permits assessment of the efficacy of the current design approaches, including the relative success of accounting for reduced spring stiffness or patterns of imposed deformation.
\end{abstract}

KEYWORDS: buried structures; earthquakes; ground movements; soil/structure interaction

\section{INTRODUCTION}

Pipelines normally operate under a combined loading environment including external pressures due to backfills and surface live loads, and internal pressures exerted by the fluids being transported. Some extreme cases pose greater threats to the pipe network, such as ground strains induced by seismic waves, and various types of permanent ground deformations. Surface faulting, landslides, seismic settlement and lateral spreading induced by soil liquefaction all feature examples of irrecoverable ruptures that jeopardise the integrity of pipelines. Among these seismic hazards, post-earthquake reconnaissance showed that ground displacement discontinuities produce higher damage rates in pipes than wave propagation (O'Rourke \& Liu, 2012). This paper focuses on the performance of pipelines experiencing soil movements within a relatively narrow zone due to offset of two portions of the earth's crust induced by surface faulting.

Soil-pipe interaction depends on the relative pipe-soil stiffness. The normal design approach for considering the effects of differential ground motion relies on beam-on-spring analysis to model the pipe with the surrounding soil, using empirical non-linear springs. These springs were developed from large-scale pulling tests or numerical simulations for steel (i.e. high stiffness) pipes (ALA, 2001; Jung et al., 2013a, 2013b). Recent studies have shown that they can provide effective estimates for the strikeslip fault-pipeline interaction problem (Xie et al., 2011), but may not be appropriate for calculating peak responses for flexible pipes (e.g. flexible pipes are defined here as those that can deflect more than $2 \%$ without significant structural distress (Moore, 2001; Gabriel, 2011)) subject to dip-slip

Manuscript received 23 June 2016; revised manuscript accepted 16 March 2017. Published online ahead of print 8 May 2017.

Discussion on this paper closes on 1 June 2018, for further details see p. ii.

* GeoEngineering Centre at Queen's-RMC, Department of Civil

Engineering, Queen's University, Kingston, ON, Canada. fault rupture (Xie et al., 2013; Saiyar et al., 2016). Erroneous analyses using empirical soil springs were obtained even for semi-rigid ductile iron pipes experiencing reverse faulting action, and a modification to derived springs was needed to obtain improved results (Erami et al., 2015). All existing analytical models for continuous pipelines within faulting zones as reported in the literature (Wang \& Yeh, 1985; Takada et al., 2001; Karamitros et al., 2007, 2011; Trifonov \& Cherniy, 2010, 2012; Wang et al., 2011; Li et al., 2012b; Kouretzis et al., 2015) follow the original assumptions of Newmark \& Hall (1975) and Kennedy et al. (1977), and employ soil springs to solve the compatibility problem using beam-on-elastic-foundation and elastic-beam theory. Significantly, these methods were all calibrated for steel pipes.

Centrifuge modelling has been used to study reduced-scale flexible pipe models subject to enhanced gravity crossing strike-slip faults (Choo et al., 2007; Ha et al., 2008b, 2010; Abdoun et al., 2009), reverse faults (Bransby et al., 2007) and normal faults (Ha et al., 2008a; Saiyar et al., 2011, 2016). A $1 \boldsymbol{g}$ shaking table apparatus was used to evaluate flexible gas pipelines under simultaneous seismic wave and strike-slip (Sim et al., 2012a) or reverse faults (Sim et al., 2012b). However, field and laboratory testing data for flexible pipes under fault rupture are scarce, and this hinders the calibration of analytical solutions or numerical models for those flexible structures. To date, in the literature, O'Rourke et al. (2008) reported the only prototype-scale tests on high-density polyethylene (HDPE) pipes under strike-slip faults; Li et al. (2012a) performed the only in-situ experiments on HDPE pipes straddling a reverse fault; and Wham et al. (2016) conducted full-scale testing of flexible, molecularly oriented polyvinyl chloride (PVCO) pipeline subjected to strike-slip faulting.

Given this relative lack of attention to flexible pipes, the aim of the current paper is to provide large-scale testing data for flexible PVC pipes experiencing a normal fault with a dip angle of $90^{\circ}$. A new fault simulator was designed where one half of the soil block can drop to model a normal fault. Two measuring systems were used to capture the pipe's elongation and flexure during faulting action, namely strain gauges and 
fibre optic sensors. Distributed sensing of strains using fibre optics has potential advantages over strain gauges, such as: (a) it is more likely to capture peak strain values; $(b)$ it is easier and faster to install; $(c)$ although the initial cost for the fibre optic analyser is high, fibre itself has low cost, which means that pipes can be tested to failure (i.e. it is expensive to conduct destructive tests on pipes instrumented with conventional strain gauges). Owing to the high cost of sample preparation, a 4 inch (nominal pipe size of 4.5 inch with an outside diameter of $122 \mathrm{~mm}$ ) PVC pipe instrumented with conventional strain gauges by Cholewa et al. (2009) was reused in this study $(1 \mathrm{inch}=2.54 \mathrm{~cm})$. The measured discrete readings along the 4 inch pipe then provide evidence for evaluating the effectiveness of fibre optic strain measurements of distributed strain along a second 4 inch dia. pipe. Upon the successful replication of those strain measurements using conventional and optical fibre systems, two smallerdiameter (1 and 2 inch) pipe tests were conducted using fibre optics to investigate the influence of fault offset, burial depth ratio and pipe diameter. A comparison between these large-scale test data and conventional design analyses based on empirical and modified soil springs (by reducing the peak force in vertical bearing springs) using different fault rupture profiles was then undertaken. The efficacy of the Winkler-based numerical analysis (ALA, 2001) and the three-beam model (Karamitros et al., 2011) was evaluated, to assess whether the analyses provide reasonable estimates of peak bending strains for flexible pipelines under normal faulting.

\section{EXPERIMENTAL SET-UP Apparatus}

A new large-scale split-box test apparatus as shown in Figs 1 and 2 has been assembled in one half of the large-scale buried infrastructure test pit within the GeoEngineering Laboratory at Queen's University. This permits evaluation of the flexural response of pipes up to $7 \cdot 1 \mathrm{~m}$ long. The split-box consists of a moving floor $3.65 \mathrm{~m}$ long and a stationary floor $3.45 \mathrm{~m}$ long, to model normal fault rupture that occurs in the bedrock stratum and propagates up through an overlying soil layer to the ground surface. As the fault propagates, the pipe responds differently on each side of the fault, as the pipe segment on the footwall side is pushed downwards into the underlying soil (i.e. the bearing side) and the pipe on the hanging wall side is pulled upwards against the overlying soil (i.e. the uplift side).
Based on the subgrade reaction method, Bransby et al. (2007) proposed a critical pipe length $l_{\mathrm{c}}$ beyond which the pipe behaves as if it is infinitely long (i.e. it is fully flexible).

$$
l_{\mathrm{c}}=4\left(\frac{E_{\mathrm{p}} I_{\mathrm{p}}}{k_{\mathrm{u}}}\right)^{0.25}
$$

where $E_{\mathrm{p}} I_{\mathrm{p}}$ is the flexural rigidity of pipe. $k_{\mathrm{u}}$ is the soil subgrade reaction stiffness and defined by $k_{\mathrm{u}}=Q_{\mathrm{u}} / \Delta q_{\mathrm{u}}$. This facilitates assessment of the length scale of a testing chamber to avoid boundary effects associated with pipe end fixity conditions. Here, the subgrade reaction curve for the soil on the uplift side is used to estimate $k_{\mathrm{u}}$. The peak resistance $Q_{\mathrm{u}}$ and the corresponding mobilising displacement $\Delta q_{\mathrm{u}}$ for dense sand are given following Bransby et al. (2007) as follows

$$
\begin{aligned}
& Q_{\mathrm{u}}=\gamma H\left(1+f_{\mathrm{d}} H / D\right) \\
& \Delta q_{\mathrm{u}}=0.02 H
\end{aligned}
$$

In the above expressions, $\gamma$ represents the unit weight of the soil, and $H$ and $D$ are the cover soil depth and the outside diameter of the pipe, respectively. The parameter $f_{\mathrm{d}}$ is defined as the uplift factor, which is used to modify the geometry of the mobilised soil wedge above the pipe; $f_{\mathrm{d}}$ has unit value for dense sand (Bransby et al., 2007).

For the 4 inch PVC pipe in soil, the subgrade reaction method indicates a length scale of $3 \mathrm{~m}$. This is supported by observations made in centrifuge tests on similarly flexible pipes (Saiyar et al., 2016), where it was demonstrated that curvatures vanish beyond a prototype-scale distance approximately $3 \mathrm{~m}$ away from the point of contraflexure for flexible pipes, and a shift of this point of contraflexure by roughly $0.1 \mathrm{~m}$ over the moving floor. A pipe $6 \mathrm{~m}$ long with a length of $3.5 \mathrm{~m}$ placed over the hanging wall was therefore chosen to accommodate the horizontal shift of the shear band from the fault plane. This conforms to the instrumentation of the 4 inch PVC pipe (Cholewa et al., 2009), so more strain gauges were located near the fault plane. Design and construction of a testing chamber $7 \cdot 1 \mathrm{~m}$ long was therefore judged sufficient to capture the curvature distribution along the pipe, without adverse effects from the end conditions with respect to flexure (although the impact of boundary conditions on axial response is different).

The test box (apparatus) also needs to be wide enough to accommodate the pipe's upward motion without undue
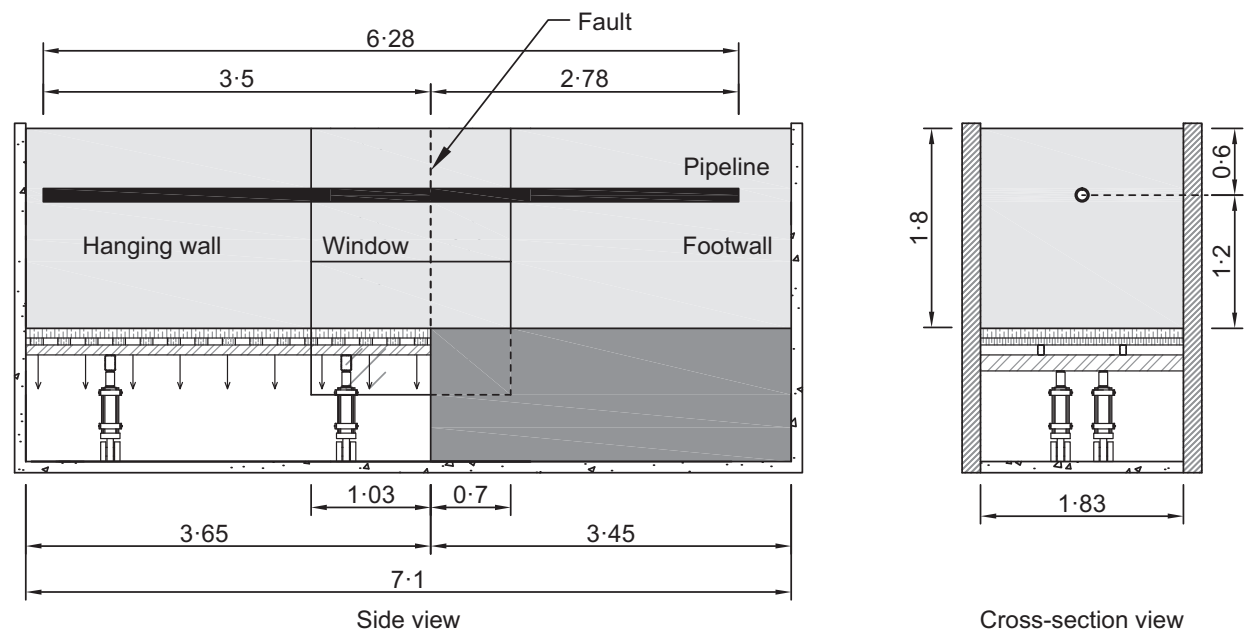

Cross-section view

Fig. 1. Schematic diagrams of the test configuration (unit: $\mathrm{m}$ ) illustrating 4 inch dia. PVC pipe instrumented with fibre optic sensors. Other pipes tested have slight variations in pipe length, but were placed to maintain the $3.5 \mathrm{~m}$ distance to the fault on the hanging wall side of the split-box 


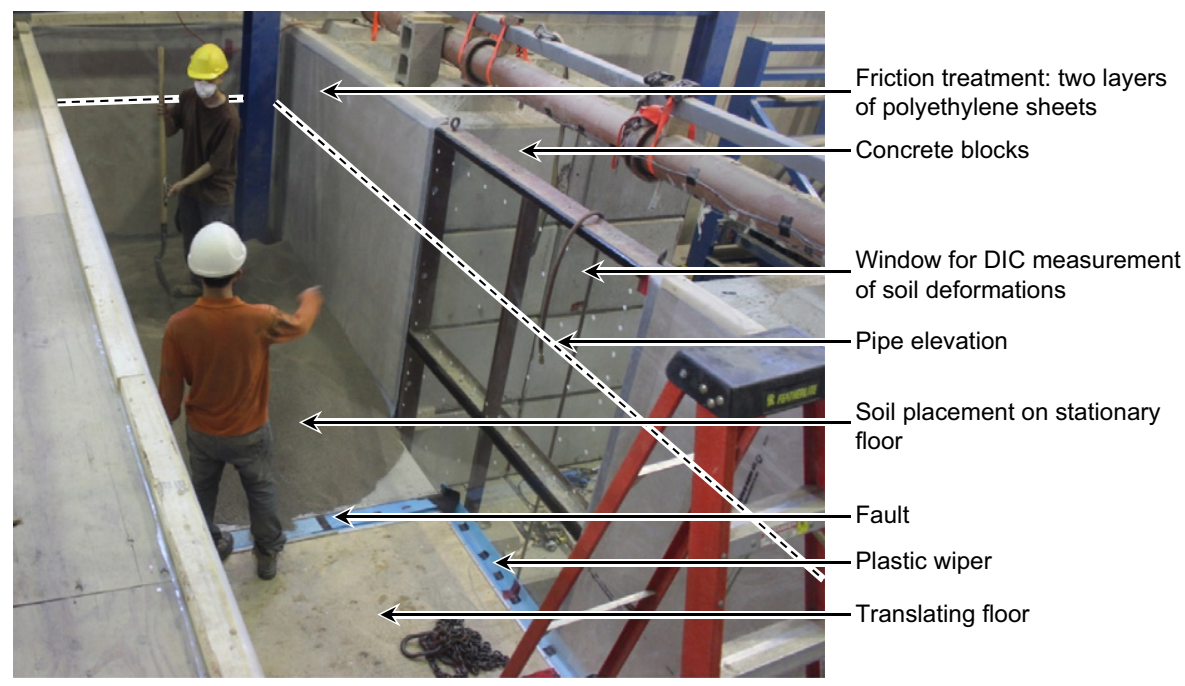

Fig. 2. Annotated photograph of test apparatus prior to backfilling

interference from the sidewalls. Centrifuge tests for pipes undergoing uplift in dense sand indicate that the disturbance could spread across an arc width of $16-18^{\circ}$ from the vertical plane (White et al., 2001; Cheuk et al., 2008). The inclination of shear planes was found to favour a kinematic arrangement that an inverted trapezoidal block lifts the soil above the pipe at the dilation angle to the vertical (White et al., 2008). Assuming an inclination angle of $20^{\circ}$ and pipe burial depth of $0.6 \mathrm{~m}$ (the choice of burial depth will be explained later), a conservative estimate of width at the ground surface is $0.56 \mathrm{~m}$. Considering potential application of this split-box testing apparatus to other projects, a width of $1.83 \mathrm{~m}$ was chosen, which is more than sufficient for this PVC pipe study.

Use of friction treatment on the sidewalls and end-walls of the split-box helps reduce the shear stress mobilised by the soil on the vertical walls, so that it better represents a soil volume of infinite width. First, a polyethylene sheet was fixed on top of each wall using lumber strips, which acted as a base layer for a second sheet to slide against. Between these two sheets, a thin film of silicone-based bearing grease was used as a lubricant. The second layer extended approximately $10 \mathrm{~cm}$ beyond the wall onto the floor below the backfill soil to avoid loss of sand around the edges. This arrangement is expected to yield a peak angle of friction of no more than $5^{\circ}$ (Tognon et al., 1999), a substantial reduction below the $20^{\circ}$ value that would otherwise be expected to occur if the backfill were moving down in direct contact with the vertical walls. One sidewall of the split-box features viewing windows, for capture of high-quality digital images for use in post-test digital image correlation analysis for the free-field soil movement. Burnett (2015) provides details of the design of the viewing windows, and indicates that the soil-glass friction on these windows is expected to be $12^{\circ}$. Further details regarding the windows of the test box are given in a subsequent section. Other design considerations for the split-box test apparatus are reported by Poon (2015).

\section{Geometry of the tests}

The geometric configuration of the tests was designed to place the pipe at a depth of $0.6 \mathrm{~m}$ below the ground surface, which falls within the practical range of $0 \cdot 3-1 \cdot 5 \mathrm{~m}$ typical of gas pipes (Groves \& Wijewickreme, 2013). They explain that this can effectively avoid the occurrence of pipe buckling, as well as prevent exposure to ultraviolet radiation and construction damage. Different diameter pipes were tested to examine the effect of burial depth ratio $(H / D)$ as the magnitude of fault offset increased. Table 1 summarises all of the tests performed.

The total soil depth of the split-box is $1.8 \mathrm{~m}$. For burial depth of $0.6 \mathrm{~m}$, this means that a depth of $1.2 \mathrm{~m}$ is left from the pipe centreline to the base of the split-box.

\section{Test pipes and backfill soils}

Saiyar et al. (2016) tested polycarbonate and acrylic model pipes in a centrifuge to investigate the flexural response of PVC pipes under normal faulting conditions. One objective of the testing programme reported in this paper is to provide full-scale experimental evidence for similarly flexible PVC pipes. The properties of the pipes selected are tabulated in Table 1.

Synthetic olivine sand has a subangular to angular shape with a gritty texture. Its inherent variation in grain colour leads to excellent texture in digital images, which is an advantage when using the software geoPIV (White et al., 2003) to calculate the deformation of the soils from digital photographs, because there is no need to artificially dye the soils with colours to obtain texture for tracking by the digital image correlation (DIC) technique. The toughness of this material (it is commercially available for use in sand blasting) also makes it suitable for use in repeated tests, where the reversals of compaction and removal do not significantly change its particle distribution.

A sieve analysis was conducted to measure the particle distribution of the material; it was then classified using the Unified Soil Classification System. A poorly graded sand, it has coefficient of curvature $C_{\mathrm{c}}$ of 0.96 and uniformity coefficient $C_{\mathrm{u}}$ of $2 \cdot 21$. It features an effective particle size $d_{10}$ of $0.46 \mathrm{~mm}$, an average particle size $d_{50}$ of $0.83 \mathrm{~mm}$ and a specific gravity of $3 \cdot 2$, which is higher than typical values for conventional silica sands. The minimum and maximum index density tests have also been undertaken to obtain the minimum and maximum densities of 1500 and $1677 \mathrm{~kg} / \mathrm{m}^{3}$ (maximum and minimum void ratios of $1 \cdot 13$ and $0 \cdot 91$ ), respectively.

Dry sand backfill was placed by releasing the sand from an overhead hopper operated from a gantry crane. Hand tamping was conducted for every $150 \mathrm{~mm}$ lift to reach the target relative density of about $55-65 \%$. Drained triaxial tests provided all the soil parameter estimates of concern to capture the stress-strain-strength and deformation 


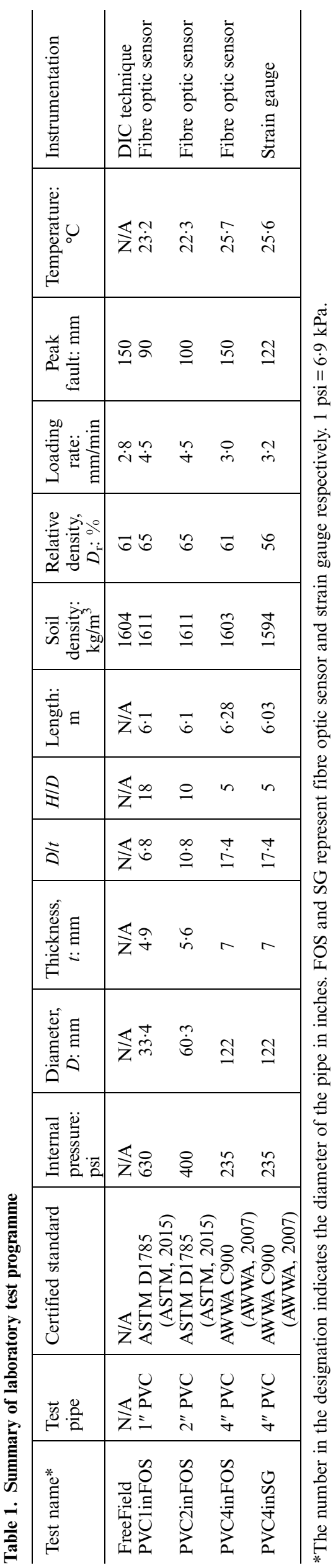

characteristics at low levels of overburden and confining stresses at the pipe burial depth: peak friction angle of $42^{\circ}$, constant volume friction angle of $34^{\circ}$, dilation angle of $16^{\circ}$, Young's modulus of $2 \mathrm{MPa}$ at geostatic stresses corresponding to the pipe burial depth and Poisson ratio of $0 \cdot 3$.

\section{Instrumentation}

Two types of measuring sensors were adopted to monitor pipe responses to fault offset. One of the 4 inch pipes was instrumented with dual foil strain gauges in pairs at 19 separate locations on the crown and invert. They all feature a gauge length of $5 \mathrm{~mm}$ with a measuring accuracy of 10 microstrain (i.e. uniaxial Showa N11-FA-5-120-11 gauges and Showa N32-FA-5-120-11 rosette gauges (Cholewa et al., 2009)). The average of longitudinal strains at these opposite sides of the pipe can be compared with axial strains measured at the springline to validate the effectiveness of using EulerBernoulli beam theory to interpret testing results. Bending strains can then be calculated as one-half the difference in strain readings between the crown and invert. A modification factor of 1.3 needs to be applied to scale up the measured strains due to local gauge hardening, as the stiffness of the strain gauge and adhesive is very close to that of the flexible PVC pipe (Cholewa et al., 2009). Subsequent comparisons of these readings with those from optical fibres confirmed the effectiveness of this calibration. Baseline readings were taken just prior to the commencement of the test.

The second 4 inch pipe was instrumented with fibre optic sensors to compare distributed measurements with discrete strain gauge readings. An optical backscatter reflectometer (OBR) 4600 system from Luna Technologies (2011) was used to provide measurements within the fibres. The backscattered light in the fibre is analysed to obtain the spectrum measurement for the purpose of comparison, so strains can then be interpreted from the correlation with the changes in spectrum (Agrawal, 2006). It has an excellent resolution of 1 microstrain over a $5 \mathrm{~mm}$ gauge length. The fibre optic measuring technique has been successfully applied to concrete pipes and HDPE pipes (Simpson et al., 2015), which demonstrated its advantages: ease of installation, scanning density and cost-effectiveness (nylon-coated fibre of US $\$ 0 \cdot 15 / \mathrm{m}$ as opposed to strain gauge cost of US $\$ 10-30 /$ gauge). The pipe surface was cleaned using sandpaper and alcohol, against which the fibres were bonded with a cyanoacrylate adhesive (Loctite 401) and then covered with a silicone sealant. It is critical to choose an appropriate spatial resolution, as it affects the measurement accuracy. This study follows previous experience (Simpson et al., 2015) to set a gauge length of $20 \mathrm{~mm}$ with a spacing of $20 \mathrm{~mm}$ along the fibre. An initial scan of backscattered light profile was conducted after backfilling of the trench was complete, which acted as a reference to be compared with subsequent scans during the test. The OBR system only measures interdependent temperature and strain. A separate measurement of temperature around the pipe is imperative to isolate the strain changes induced by the temperature effect, even though the tests reported here were conducted over short time duration in a temperature-controlled room. Furthermore, plastics are sensitive to temperature variations and it makes sense to have thermocouples attached to the PVC test pipes. Two smaller-diameter (1 and 2 inch) pipes were instrumented by fibre optics alone. Fig. 3 depicts the plan view of all four instrumented pipes, as listed in Table 1.

Two string potentiometers were mounted under the moving floor between the two north jacks (SP-1) and the two south jacks (SP-2). The north jacks are controlled by one drive shaft and the south jacks are controlled by another set. 


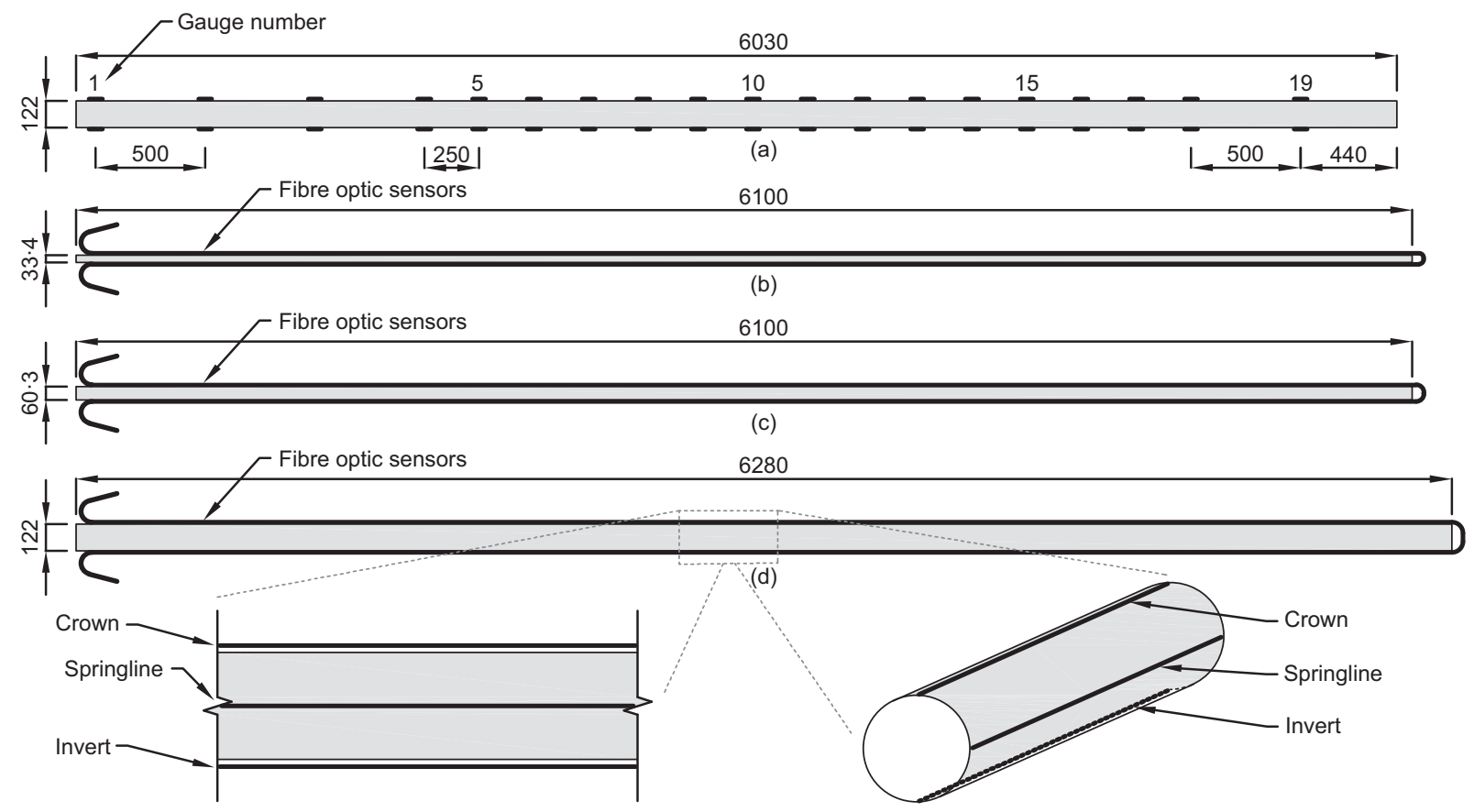

Fig. 3. Schematic diagrams of instrumented pipes used in experiments: (a) 4 inch pipe instrumented with strain gauges; (b) 1 inch pipe instrumented with fibre optic sensors; (c) 2 inch pipe instrumented with fibre optic sensors; (d) 4 inch pipe instrumented with fibre optic sensors (unit: mm)

String potentiometers are used to monitor each location. This permits control of floor displacement in real time during the test, with screw jacks turned at identical rates to lower the whole floor downwards to effect rigid body movement.

Vertical displacements on the ground surface were captured using a servo-controlled total station to track the movements of 14 discrete surveying prisms placed $0.25 \mathrm{~m}$ apart, in proximity to the fault plane. This provides an accuracy of the surface displacements within $0.3 \mathrm{~mm}$. Two $15.8 \mathrm{~mm}$ thick transparent windows having dimensions of $1.83 \mathrm{~m}$ by $1.22 \mathrm{~m}$ were stacked together to enable observation of soil deformations during fault rupture propagation and post-test analysis using the DIC technique. Image capture software DSLR Remote Pro Multi-Camera synchronised the settings of two Canon EOS Rebel T4i DSLR cameras to capture image pairs (one for the top and one for the bottom window) at $10 \mathrm{~s}$ intervals. Each camera was adjusted to have the longest focal length of $55 \mathrm{~mm}$ and positioned on a tripod at a height corresponding to the centre of each visible window. This can effectively reduce the measurement error induced by lens distortion (Take, 2015). The windows were specially designed to conform to the size of the photograph. A very conservative estimate of the precision of DIC measurements is approximately $0 \cdot 1$ pixels (White et al., 2003; Stanier et al., 2015). In this experiment, the cameras can produce images with a resolution of 3456 by 2304 pixels. This indicates that the size of each pixel would represent $1.83 \mathrm{~m} / 3456$ pixels, or $0.53 \mathrm{~mm}$, and so the accuracy of the measured displacements is expected to be $0.05 \mathrm{~mm}$

\section{Testing method}

Two in-air bending tests were conducted on the 4 inch pipe instrumented with strain gauges and the 2 inch pipe instrumented with fibre optic sensors. The test configuration corresponds to a simply supported beam subjected to a concentrated load at midspan. The closed-form solution of this problem was used to back-calculate the flexural rigidity
$\left(E_{\mathrm{p}} I_{\mathrm{p}}\right)$ of the test pipes. Furthermore, this also permitted evaluation of the Young's modulus $\left(E_{\mathrm{p}}\right)$ of the PVC material used in the pipe.

In the literature, the only experiments reported to date where the normal fault-pipe interaction problem was studied are the centrifuge tests performed by Ha et al. (2008a) and Saiyar et al. (2016), where the ratios of peak fault offset to pipe diameter are 1.2 and 0.9 , respectively. In the current study, a similar fault offset of one pipe diameter was chosen for the 4 inch pipes. Smaller-diameter pipes can accommodate larger fault displacement (i.e. $2 \cdot 7 D$ for the 1 inch pipe and $1 \cdot 7 D$ for the 2 inch pipe) as the axial and bending strains are proportional to the pipe diameter. All tests were continued until failure of the optical fibre strain measurement system (indicated by bad correlation of scattered light, where a long length of fibres presented low spectral shift quality and peak strains were missed). In addition, the glass core of the nylon-coated fibre is not expected to rupture until after approximately $1 \%$ strain; this was confirmed because strain measurements up to $1 \%$ are reported in a subsequent section without fibre rupture.

Ideally, boundary conditions at the pipe ends need to account for the axial force-displacement response at those positions associated with a much longer pipeline. Use of a fixed end condition corresponds to the situation when no soil-pipe slippage occurs at the pipe ends, with axial restraining forces developing at the ends if the test pipe is too short. This pipe fixity is generally preferred in centrifuge testing as it is hard to implement more sophisticated control over the end conditions for reduced scale pipes (Choo et al., 2007; Ha et al., 2008a, 2008b, 2010; Abdoun et al., 2009; Rojhani et al., 2012). It also generates a conservative estimate of the tensile axial force (Yoshizaki et al., 2003). Alternatively, Sim et al. (2012b) assigned partial fixity: the pipe was restrained on the footwall side and free on the hanging wall side, which makes the boundary conditions more complex and perhaps unrealistic. Similar to a pipe response to nearby tunnelling (Klar et al., 2005, 2008; Vorster et al., 2005; Marshall et al., 2010), flexural behaviour 
dominates for the normal fault-pipeline interaction problem. It was consequently decided to leave the pipe ends free (i.e. not to tie the pipe ends to anchor blocks). This means that axial force remains zero at the ends, even at high values of fault rupture. The advantage of the free end condition is that there is no additional restraint against bending due to axial tensions that may arise from the fixity, so that the test should produce conservative evaluations of pipe bending. Alternatively, this set of tests could be considered as corresponding to a scenario involving $6 \mathrm{~m}$ long pipe segments connected with gasketed joints, where slippage is allowed to occur so that axial forces are zero.

Bransby et al. (2007) modelled the ground fault as a quasi-static process using a step-like function with a sufficiently slow loading rate. They indicated that the influence of near-field ground velocity of the seismic ground motion on the fault rupture propagation through the soil layer is negligible. Modelling the seismic waves and the fault action simultaneously can reduce the pipe strains, as the ground shaking lowers the soil shear strength (i.e. resistance) and leads to a reduction in the soil-pipe interaction forces (Sim et al., 2012a). The seismic wave can therefore be eliminated as a variable (the inertia effects of the pipeline are ignored), and the relatively slow imposition of the differential ground movement should produce higher measurements of demand on the pipelines. The loading rate is not the same in all the tests owing to manual operation of the fibre scanning. However, it will be shown subsequently that, for the quasi-static conditions used to examine buried pipes under differential ground motion, the loading rate did not influence the strain measurements (two pipes with the same diameter were tested at different load rates, but exhibited essentially the same responses). This is consistent with the observation of Abdoun et al. (2009) that the effect of slow fault offset rates on the magnitudes and locations of the peak strains will be insignificant.

\section{EXPERIMENTAL RESULTS}

In-air bending tests

Flexible pipes made of polymer materials often feature complex constitutive behaviour that can be highly non-linear and time dependent. However, unlike polyethylene, PVC can exhibit linear elastic behaviour up to $3 \%$ strain (Pixa et al., 1988; Povolo et al., 1996; Wham et al., 2016). As the PVC modulus is a function of polymer crystallinity and density, it is valuable to perform in-air bending tests to determine the flexural rigidities of the test pipes. The closed-form solution of a simply supported beam with a concentrated load, $F$, acting at the midspan produces the maximum bending strain as follows

$$
\varepsilon_{\mathrm{b}, \max }=\frac{F l}{4 E_{\mathrm{p}} I_{\mathrm{p}}} \frac{D}{2}
$$

where $l$ represents the span length between two roller supports.

The comparison of bending strains measured from laboratory tests and evaluated by the closed-form solution is illustrated in Fig. 4(a) for 4 inch pipe instrumented with strain gauges under five levels of load increment. Experimental data are generally close to the closed-form solution. A back-calculation was conducted to obtain the flexural rigidity from the testing data directly, as given in Fig. 4(b), where an average of $12.5 \mathrm{kN} \mathrm{m}^{2}$ was obtained. The Young's modulus of the pipe was then determined to be $3 \mathrm{GPa}$. There is no significant stress- or strain-dependent response, as the strains were essentially proportional to the applied load. This is consistent with the observation of G'Sell \& Jonas (1981) that PVC is not as sensitive to load rate effects as polyethylene. Flexural testing of the 4 inch pipe was subsequently undertaken up to maximum bending strains of $1 \cdot 5 \%$, without evidence of creep, and with negligible levels of residual strain after unloading, providing further evidence that the pipe response can be considered linear and elastic.

Similarly, the testing results of 2 inch pipe instrumented with fibre optic sensors is depicted in Fig. 5(a), along with the analytical calculations. Fibre optics can provide more readings along the pipe which produces a much smoother distribution than the discrete strain gauge measurements as no 'gauge factor' is required to account for local stiffening. At the midspan, bending strain peaks. The fibres did not provide a distinct value of peak strain at the 'beam' midpoint, and instead there was a smooth transition between the linear distributions to the left and right of the midpoint. This may have partially resulted because the concentrated force was applied within a small area in the laboratory instead of the

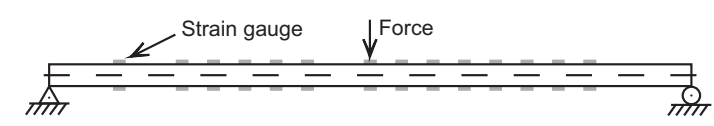

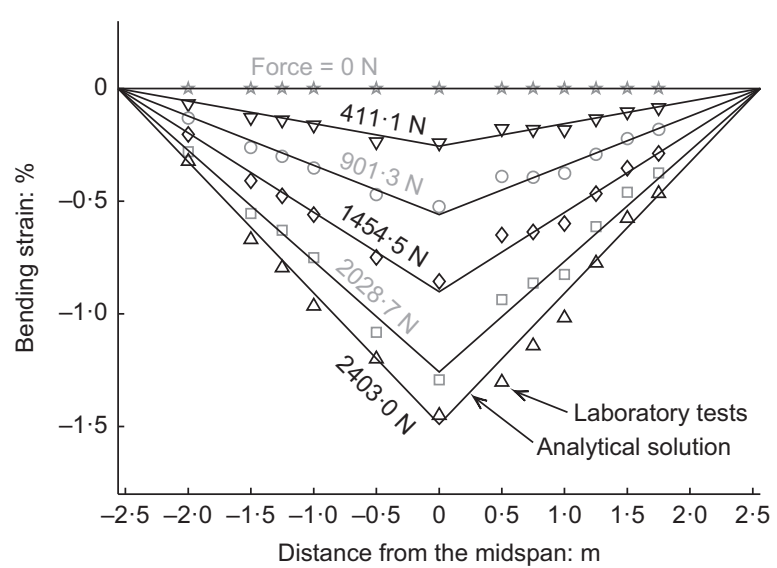

(a)

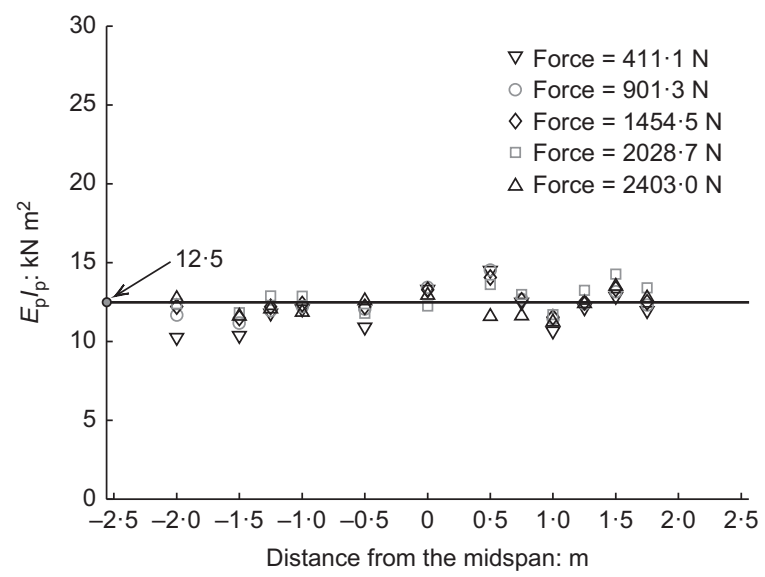

(b)

Fig. 4. In-air bending test for 4 inch pipe instrumented with strain gauges: (a) bending strain distribution; (b) derived flexural rigidity 


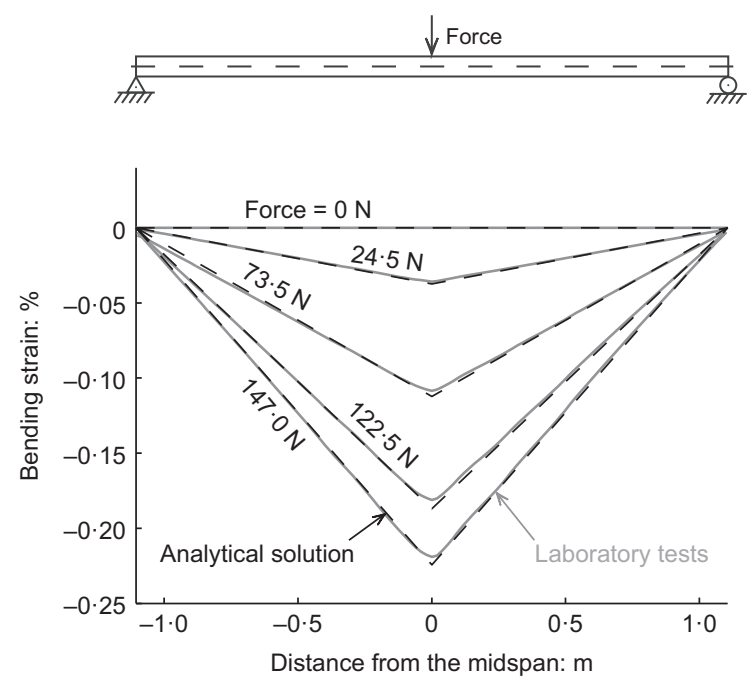

(a)

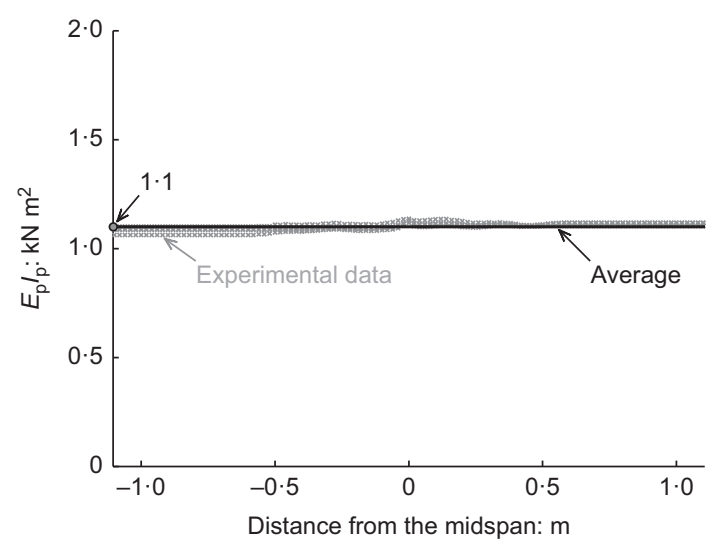

(b)

Fig. 5. In-air bending test for 2 inch pipe instrumented with fibre optic sensors: (a) bending strain distribution; (b) derived flexural rigidity

idealised point load configuration. The flexural rigidity values calculated from the test fall in a very narrow band, Fig. 5(b). This also demonstrates the advantage of fibre optic measurements compared to the conventional strain gauges. A consistent Young's modulus of $3 \mathrm{GPa}$ was also calculated. Although the flexural rigidity of the 1 inch pipe was not measured directly in an in-air bending test, the same Young's modulus of $3 \mathrm{GPa}$ was assumed and $E_{\mathrm{p}} I_{\mathrm{p}}$ was calculated using the cross-sectional geometry.

\section{Free-field soil test}

A free-field soil test was conducted first to examine the functionality of the split-box apparatus. Abdoun et al. (2009) reported negligible influence of fault offset rate on pipe response. In the current study, slow loading rates were also adopted due to hand cranking of screw jacks (rates even slower than those of Abdoun et al. (2009)). This eliminates the inertia effect, so that the fault rupture propagation is modelled as a quasi-static step-like process.

After the test, the ground surface deformations exhibited a smooth profile, Fig. 6 . At the boundaries of the hanging wall side, the soil dropped uniformly across the box, without inclined soil slopes forming against the walls. This indicates that the friction treatment was effective at reducing

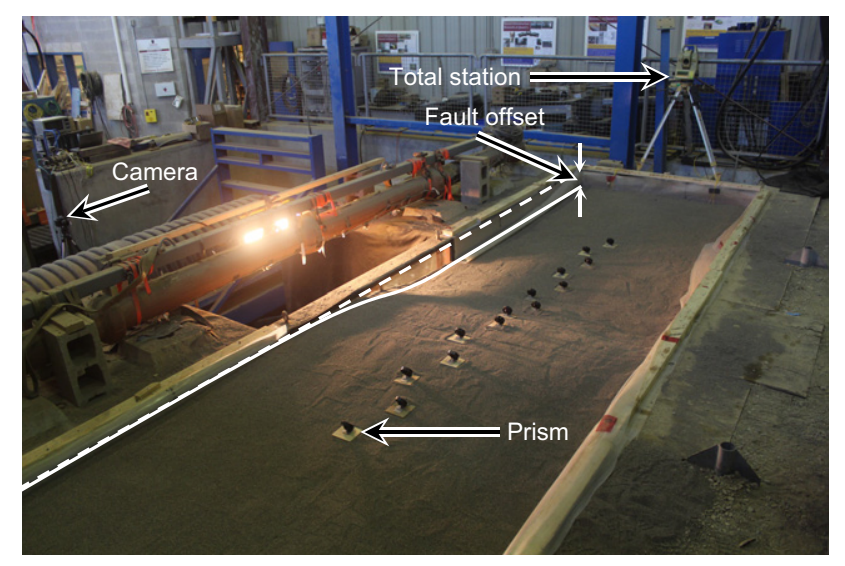

Fig. 6. Post-test ground surface deformations the influence of boundary friction on the soil behaviour. Fault offset is highlighted for the differential settlement between the initial surface elevation shown with the broken line and the post-test surface level shown with a continuous deformed solid line. The nature of dry sand is that it cannot form a sudden dislocation across the fault, but forms a slope at the ground surface with an inclination less than its constant volume friction angle. Sands at the stationary floor move in to fill any voids left by the soil dropping with the moving floor (see Fig. 7(a)). Therefore, the ground surface exhibited a continuous profile after the test. The buried pipe tests had very similar post-test ground surface deformations compared with those seen in the free-field soil test and the results are omitted here for brevity. At the opposite side of the fault plane, the mechanism reverses and the pipe on the footwall side is pushed downwards (the one on the hanging wall side is pulled upwards). The applied fault discontinuity could cause uplift failure of the overlying soil on the hanging wall side, but was not sufficient to cause cracks on the ground surface.

Images captured through the windows using cameras were subsequently analysed. The displacement field shows the vectors for all tracking patches that were displaced from the original state to the final fault offset, Fig. 7(a). Rotation of arrows indicates the inclination of the shear band, and a more obvious illustration is given in Fig. 7(b) based on vertical soil displacement contours. Extensive ground discontinuity concentrates near the 'bedrock stratum' (the floor of the test box) and spreads over an increasingly wider area as it approaches the ground surface. At a given elevation, discrete soil displacements from DIC analysis can be interpreted using a continuous mathematical model, such as error (Saiyar, 2011) or logistic function (Ni, 2016). The length scale from the location of peak curvature to the point of contraflexure is defined as $i_{\text {soil }}$, Fig. 7(c). Analogous notation is used for the pipe kinematics, with $i_{\text {pipe }}$ used to characterise the pipe deflection profile. The normal fault-pipeline interaction problem is not symmetric, since the soil resistance provided to the pipe (its bearing capacity) in the hogging zone (the section of deformed pipe which is concave down) is much larger than its uplift counterpart in the sagging zone (the section of deformed pipe which is concave up) for shallow burial depth (O'Rourke \& Liu, 2012). Both the peak curvatures and length scales are therefore different for the pipe segments on either side of the fault. 


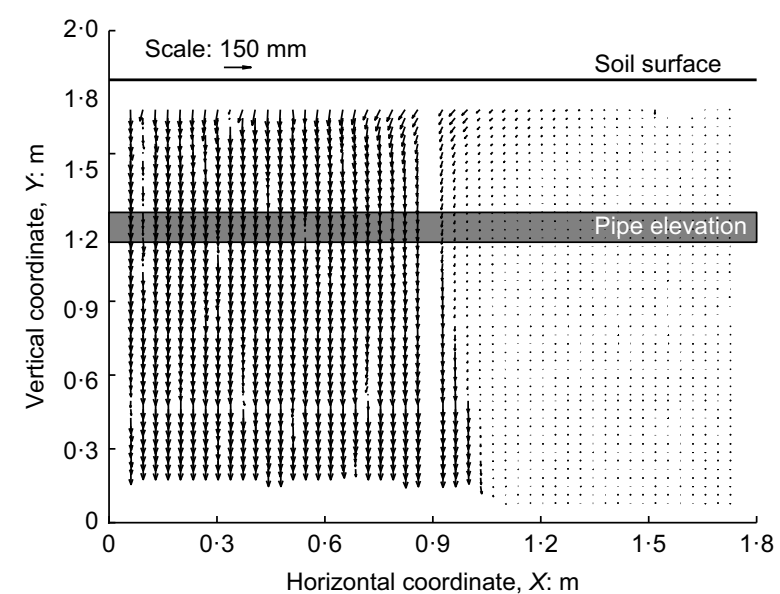

(a)

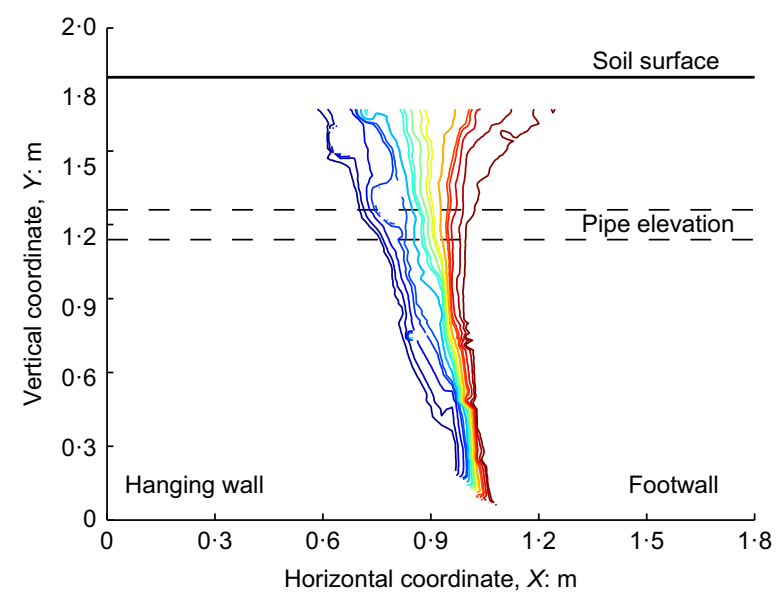

(b)

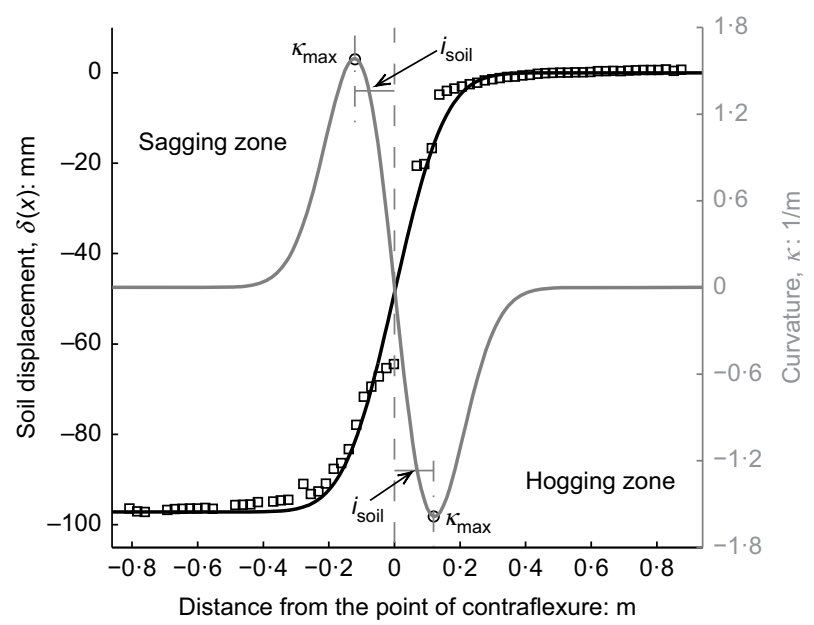

(c)

Fig. 7. Digital image correlation analysis of free-field soil test at a fault offset of $150 \mathrm{~mm}$ : (a) displacement field; (b) contours of vertical displacement illustrating location and width of shear zone; (c) derivation of curvature distribution from displacement profile

The length scale of ground surface deformations $\left(i_{\text {surf }}\right)$ obtained from DIC analysis is compared to those measured using the total station survey in Fig. 8. Ni (2016) reported that the length scale of the soil displacement is a linear function of bedding depth, $B$, from a certain elevation to the bedrock stratum (i.e. $i_{\text {soil }}=0 \cdot 18 B$ ). In this case, the soil layer thickness of $1.8 \mathrm{~m}$ produces an $i_{\text {surf }}$ value of $0.32 \mathrm{~m}$, which is similar to the flexural length scale seen in all the experimental measurements of the ground surface. The $i_{\text {surf }}$ values

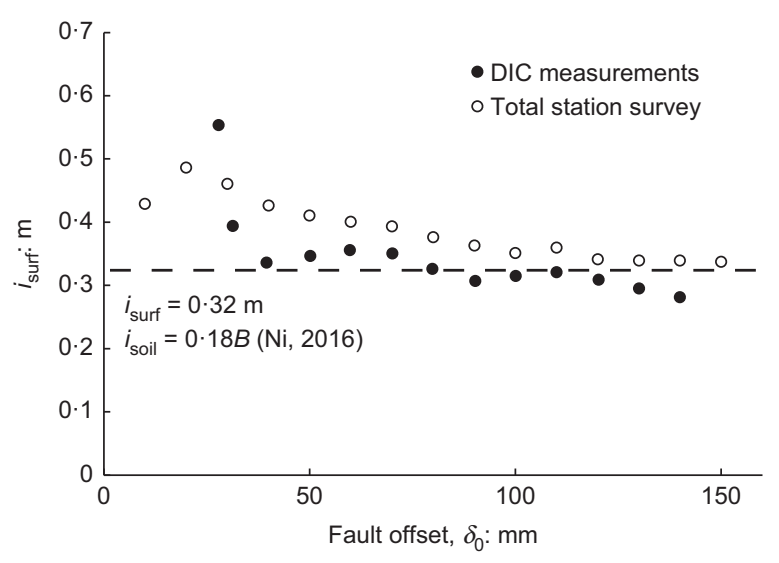

Fig. 8. Length scale of ground surface deformations obtained from DIC analysis and total station survey

calculated from the total station data are generally higher than those analysed from the DIC results, likely because the DIC analysis cannot be used to evaluate the exact profile of the ground surface because the view of the camera was obstructed by the window frame. The DIC measurements of the $i_{\text {surf }}$ values correspond to a slightly lower level (just below the ground surface), which should be smaller due to the width of soil rupture zone reducing with depth. Experience from the literature investigating settlement trough geometry arising from tunnelling in clay (Mair et al., 1993) and sands (Marshall et al., 2012) indicates that caution should be applied in extrapolating the relationship of $\mathrm{Ni}$ (2016) to other materials with differing volumetric behaviour on shearing.

\section{Pipe burial tests}

Four pipe burial tests were conducted, examining pipes with various diameters. The 4 inch pipe test has a burial depth ratio of 5 , which is very close to $H / D=5 \cdot 2$ as used in the centrifuge tests of Saiyar et al. (2016). As explained earlier, testing was conducted on two different 4 inch pipes to investigate the performance of fibre optic sensors relative to conventional strain gauges. Fig. 9 shows the measured axial and bending strains for the 4 inch pipe instrumented with strain gauges. The distribution of axial strain indicates that the testing chamber is not sufficient to accommodate the unanchored length of the pipe, which is several hundred times the pipe diameter based on calculations employing the axial response solutions of Karamitros et al. (2011). Axial slip between the soil and the pipe could initiate even at small initial values of fault offset. As discussed earlier, the choice of free end conditions for the pipe ensures that conservative measurements of bending behaviour are obtained in the experiments.

The flexural response is dominant, and it is clear that peak bending strain is about one order of magnitude higher than peak axial strain. Curvatures essentially vanish within a distance of approximately $2.15 \mathrm{~m}$ on the moving floor side and $1.25 \mathrm{~m}$ on the stationary floor side from the fault, respectively. The point of contraflexure rests over the hanging wall side, which reflects the inclination of the shear band from the vertical as it propagates up from the base and spreads out as it approaches the ground surface, Fig. 7(b).

The strains shown in Fig. 9 have been separated into axial and bending components to distinguish the effects of flexure and longitudinal stretching on the pipe strains, since the current design approach is to decompose fault actions in respective planes and examine the components in isolation. Instead, Fig. 10 presents the axial strain responses at three 


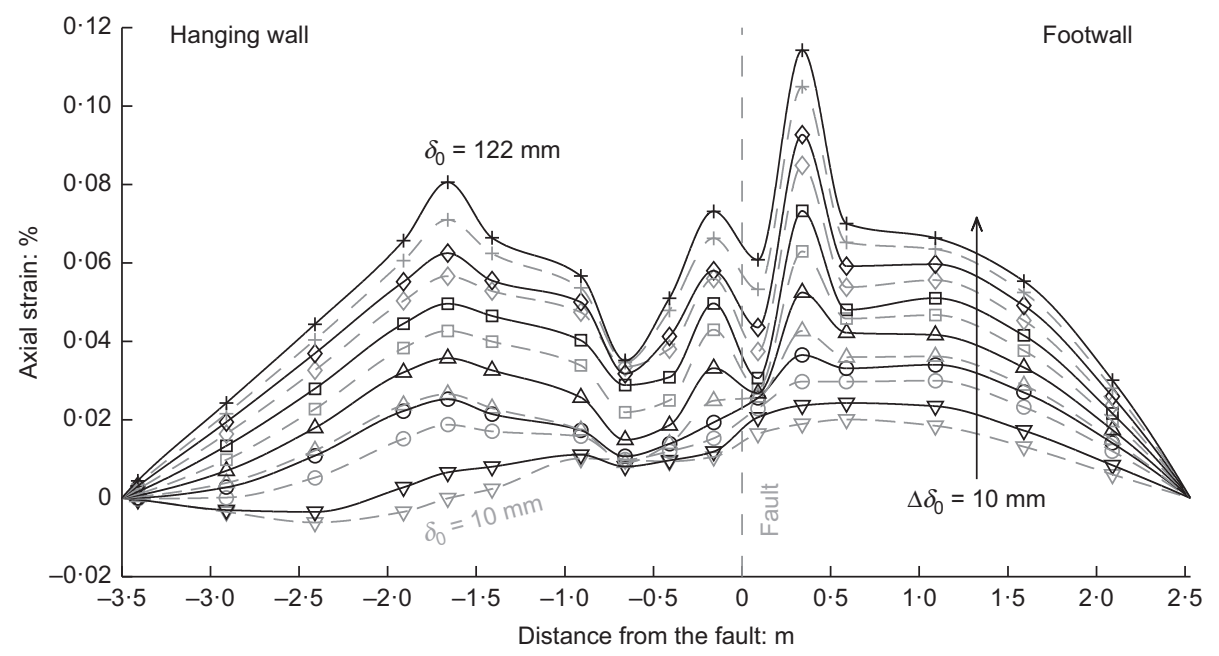

(a)

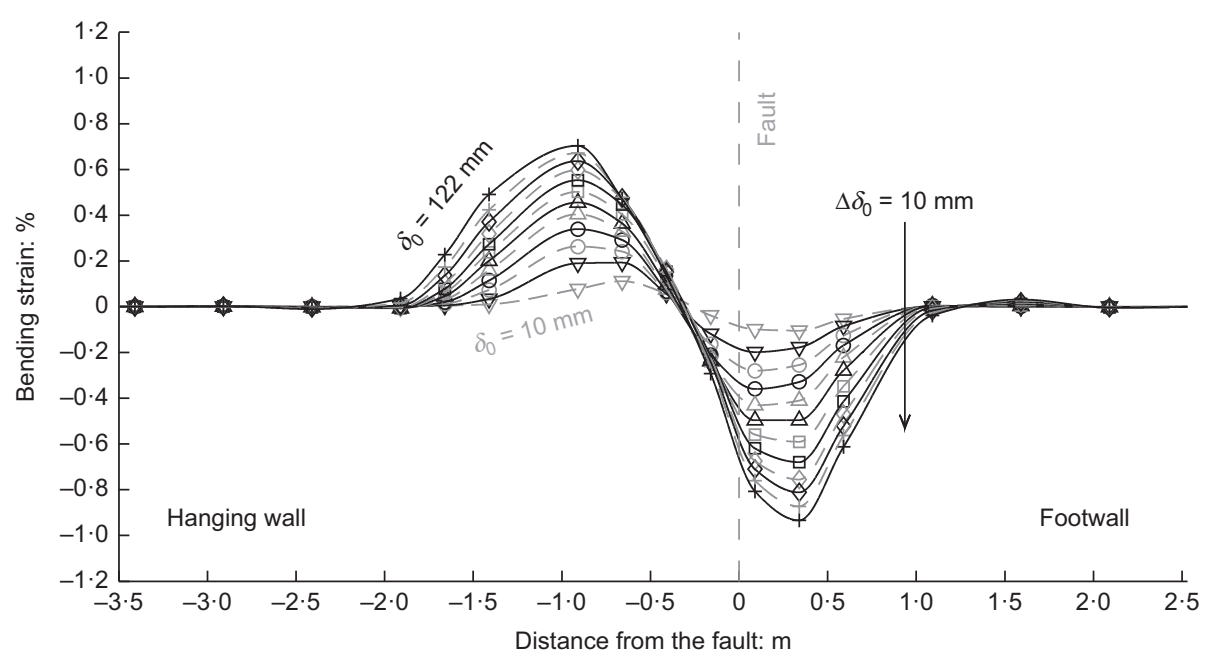

(b)

Fig. 9. Flexural responses of 4 inch pipe instrumented with strain gauges: (a) axial and (b) bending strain distribution at various offsets as functions of distance from the fault

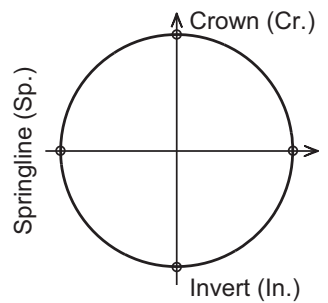

Longitudinal strain: \%

(a)

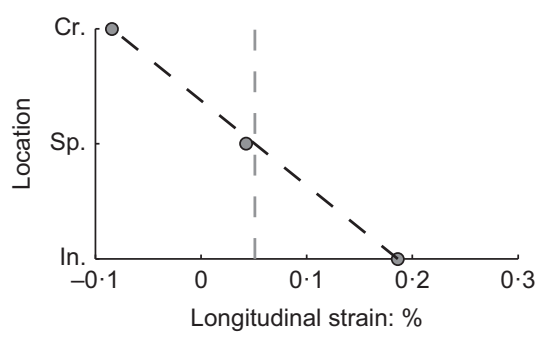

(d)

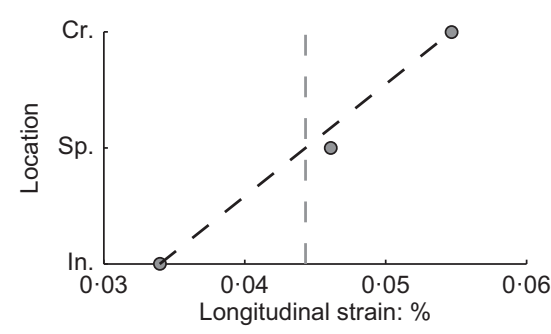

(b)

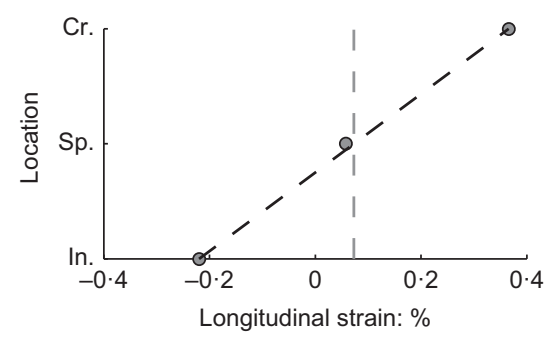

(e)

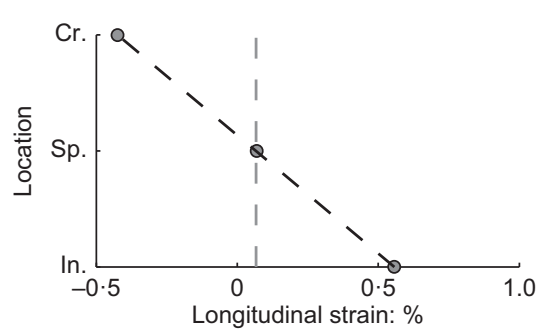

(c)

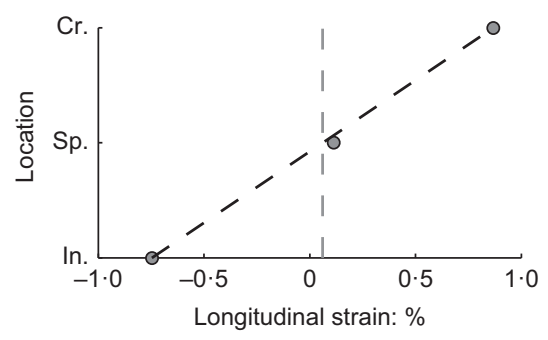

(f)

Fig. 10. Longitudinal strains at the extreme fibres of the pipe section: (a) locations of strain gauges around the cross-section; (b) at $-2 \cdot 41 \mathrm{~m}$ from the fault; (c) at $-1.41 \mathrm{~m}$ from the fault; (d) at $-0.41 \mathrm{~m}$ from the fault; (e) at $-0.16 \mathrm{~m}$ from the fault; (f) at $0.09 \mathrm{~m}$ from the fault 
points across the pipe cross-section. Strains at five different axial positions are shown in the figure, which were chosen because the measurements at pipe crown, invert and springline were available at these locations. In each location, the strain distributions vary linearly from crown to invert, suggesting that plane sections remain plane. Hence, these data confirm that thin elastic beam theory is applicable for treatment of longitudinal strains. This observation is consistent with the calculation of a relative pipe-soil stiffness of 1500 (i.e. the modulus of elasticity of the pipe and the soil is $3 \mathrm{GPa}$ and $2 \mathrm{MPa}$, respectively), which is within the range

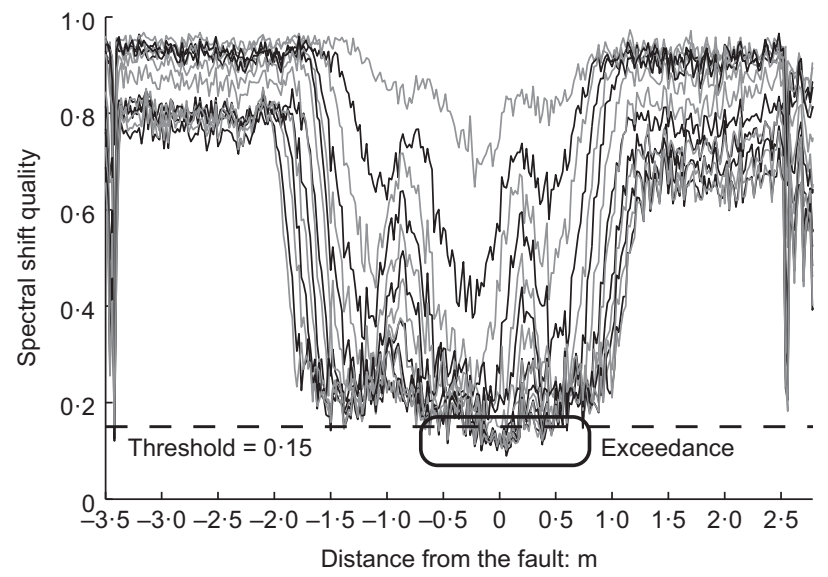

(a)

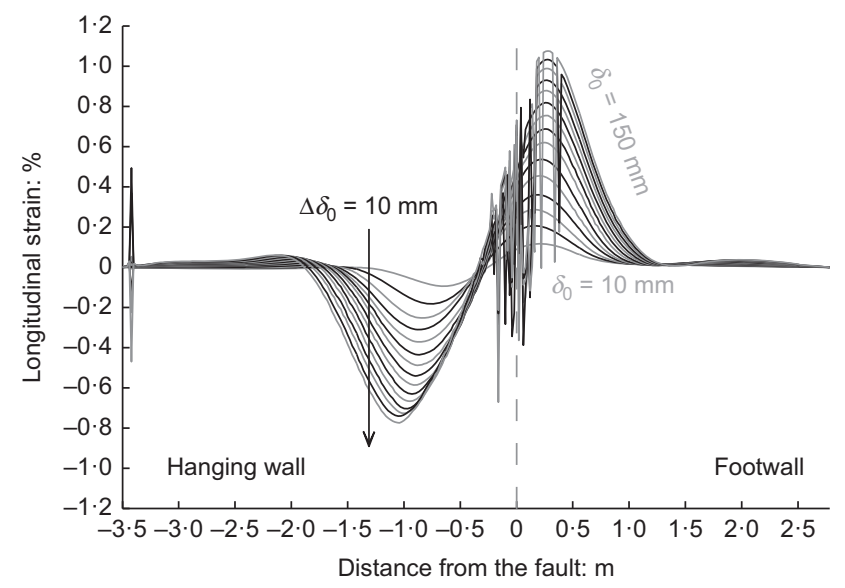

(c)

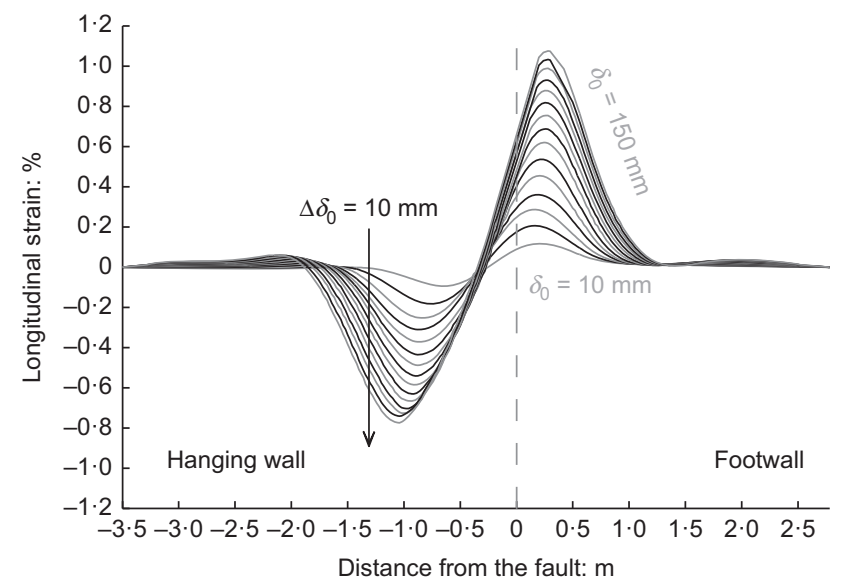

(e) reported by Klar \& Marshall (2008) in which thin elastic beam theory was found to match shell element theory.

Longitudinal strain measurements on the 4 inch pipe obtained from the fibre optic instrumentation are presented in Fig. 11, where the spectral shift quality drops significantly even at small fault offsets (especially within the zone proximal to the fault plane). When the fibre is physically stressed or kinked in that area, and if there are vibrations or movements in the patch-cord leading up to the sensing fibres, a low spectral shift quality that is below the statistical data filtering threshold of $0 \cdot 15$ is expected (due to the high strain

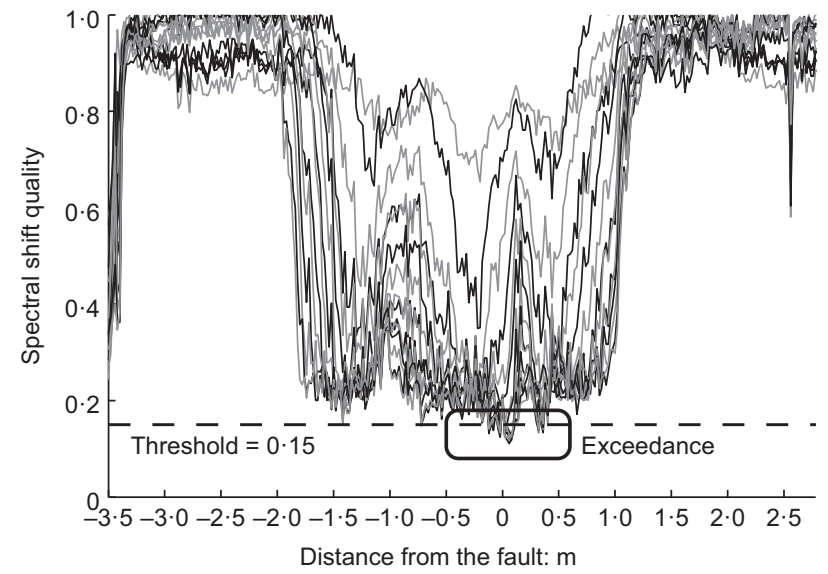

(b)

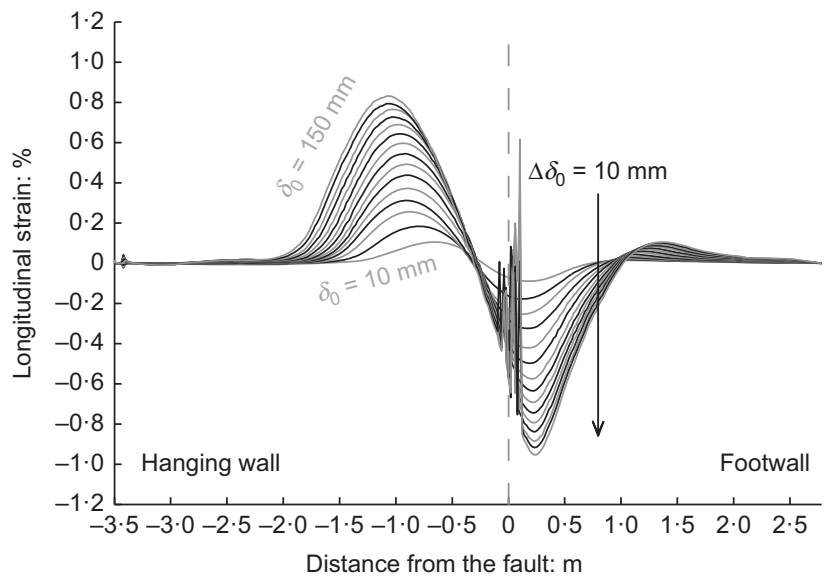

(d)

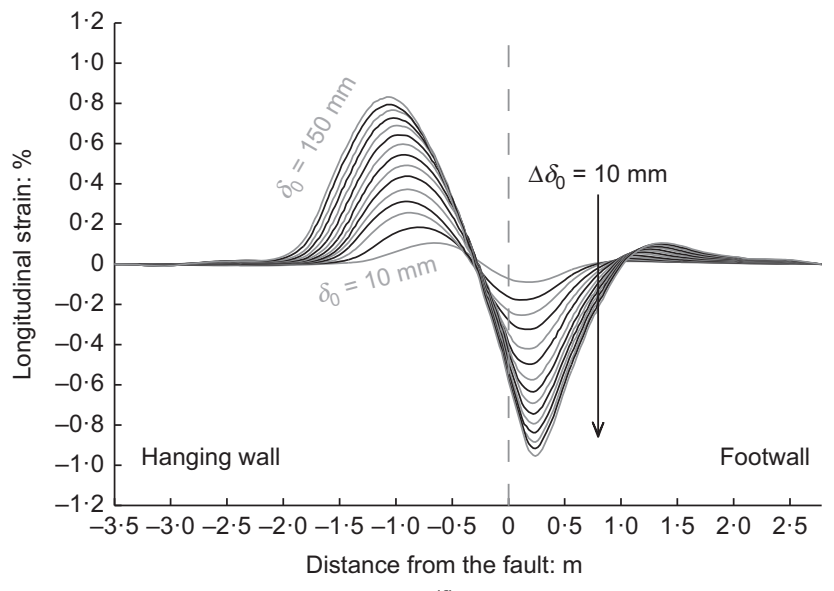

(f)

Fig. 11. Unreliable strain measurements at large strain gradients: (a) spectral shift quality at crown; (b) spectral shift quality at invert; (c) measured longitudinal strains at crown; (d) measured longitudinal strains at invert; (e) interpreted longitudinal strains at crown; (f) interpreted longitudinal strains at invert 


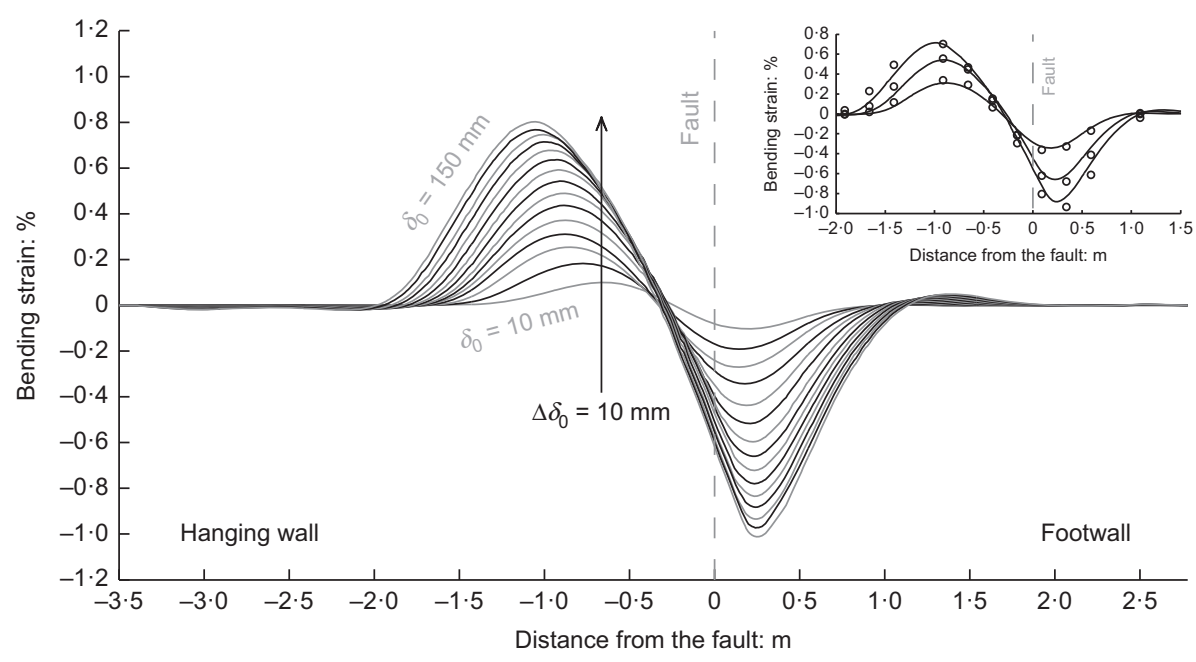

Fig. 12. Bending strain distribution of 4 inch pipe instrumented with fibre optic sensors at various offsets as functions of distance from the fault (the inserted figure shows the comparison of strain measurements obtained from fibre optic sensors in continuous lines and strain gauges in dots at fault offsets of $40 \mathrm{~mm}, 80 \mathrm{~mm}$ and $120 \mathrm{~mm}$ )

gradients). Therefore, the data in this region provide little insight into the true strain readings (Luna Technologies, 2011). Unreliable data were therefore deleted in the calculation before filtered smooth strain distribution profiles. Decomposed bending strains for the 4 inch pipe are given in Fig. 12. The general trends are very similar to the strain gauge readings in Fig. 9 (see the inserted figure in Fig. 12). Peak curvature positions are seen within $2.05 \mathrm{~m}$ of the dislocation on the hanging wall side and $1.15 \mathrm{~m}$ on the footwall floor side, respectively (these differ by about $5 \%$ and $8 \%$ for the two separate tests). At $120 \mathrm{~mm}$ fault offset, the peak bending strains evaluated in the test instrumented with fibre optics lie within $5.5 \%$ and $1.5 \%$ of values measured from the experiment using strain gauges in the hogging and sagging zones, respectively. The magnitude and length scale of the measured strains agree well and this demonstrates the repeatability of the tests. The filtering process involved deleting data with low spectral shift quality. Although some data were deleted, the fibre optic measurement system still provided accurate and reliable strain data in flexure (due to its excellent spatial resolution), which is the primary concern of this study. However, the filtering caused oscillations in the axial strains and further interpretation of the test results is needed, which will be explained later. Overall, fibres have the advantage in that they provide many more measurements, whereas strain gauges provide discrete data points that may miss the peak due to the much lower number of measurement points (even ignoring the additional advantage of easier fibre installation).

Following the success of replicating the strain distribution using the fibre optic sensors, small-diameter pipes were also tested using optical fibre measurements. Flexural responses of 2 inch and 1 inch pipes are displayed in Fig. 13. All test pipes presented double curvature bending with a larger magnitude of peak strain occurring in the hogging zone and a smaller one in the sagging zone. It is interesting that all four tests were terminated when maximum bending strains approximately equalled $1 \%$. It is an indication of the maximum strain that nylon-coated fibre can withstand and still provide meaningful measurements. Beyond that, rupture of the glass core of the fibres may occur. More localised flexural behaviour can be noticed for smaller diameter pipes, where curvatures vanish within 1.15 and $0.7 \mathrm{~m}$ for the 2 inch pipe and 0.9 and $0.55 \mathrm{~m}$ for the 1 inch pipe on the hanging wall and footwall sides, respectively.
Taking the 2 inch pipe test as an example, the measured axial strain distribution shows the oscillation between two local peaks, Fig. 14(a). This is induced by the filtering process (unreliable data identified by low spectral shift quality were deleted, in accordance with the guidelines from Luna Technologies (2011)). Linear interpolation was conducted to remove undesired variations (these interpolated regions have been identified using broken lines). All four tests were terminated at peak bending and axial strains of approximately $1 \%$ and $0 \cdot 15 \%$.

All tests show unsymmetric behaviour along the pipeline under normal faulting in both the axial and bending responses. This is caused by the nature of the problem, where one half of the pipe-soil interaction is limited by bearing capacity and the other half is controlled by uplift capacity, which is smaller (ALA, 2001). This explains why the peak bending strain on the footwall side is larger than the strains on the hanging wall side. Axial strain distribution presents a local peak at each side of the fault due to integrated shear (measured values in Fig. 14(a)), which is also in line with the peak soil reaction (i.e. normal force acting across the soil-pipe interface together with interface slip in the axial direction produces axial friction). Between two high-curvature zones, the actual relative pipe-soil displacement proximal to the fault becomes somewhat smaller than that located some distance away from the fault (Ha et al., 2008b). Axial strain then dips down at the point of contraflexure of bending response.

However, the axial strain distribution of the normal faultpipeline interaction problem can be tentatively computed by assuming the pipe experiences two block patterns of longitudinal permanent ground deformation acting in opposite directions. One can anticipate that the peak axial strain occurs proximal to the point of contraflexure on the bending strain profile and reduces thereafter with distance from the peak (Karamitros et al., 2011). The measured response of two local peaks with oscillations near the fault is questionable due to high strain gradients, which deserves further investigation. An interpreted response of the data within a bad correlation zone (i.e. broken lines) is therefore performed and illustrated in Fig. 14(b). The measured and interpreted peak values do not vary too much (e.g. at $100 \mathrm{~mm}$ fault offset, the magnitudes of peak axial strain differ by about $12.6 \%$ ) and they provide a range of axial responses that a pipe might experience at a specific fault level. The accumulation 


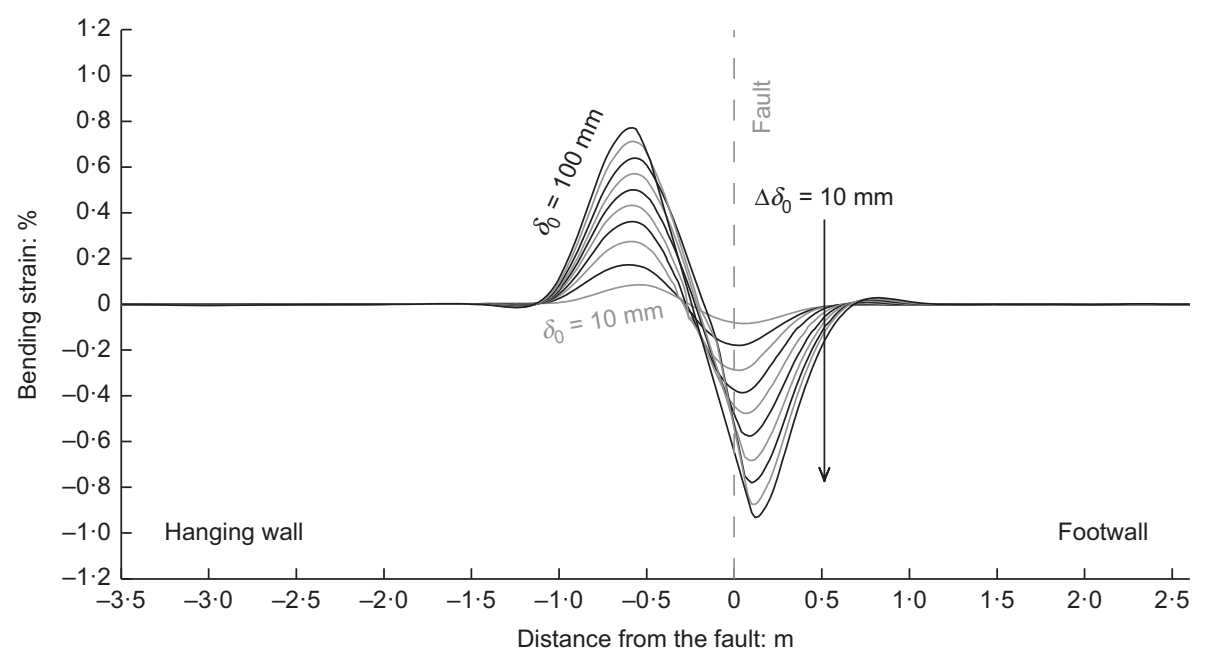

(a)

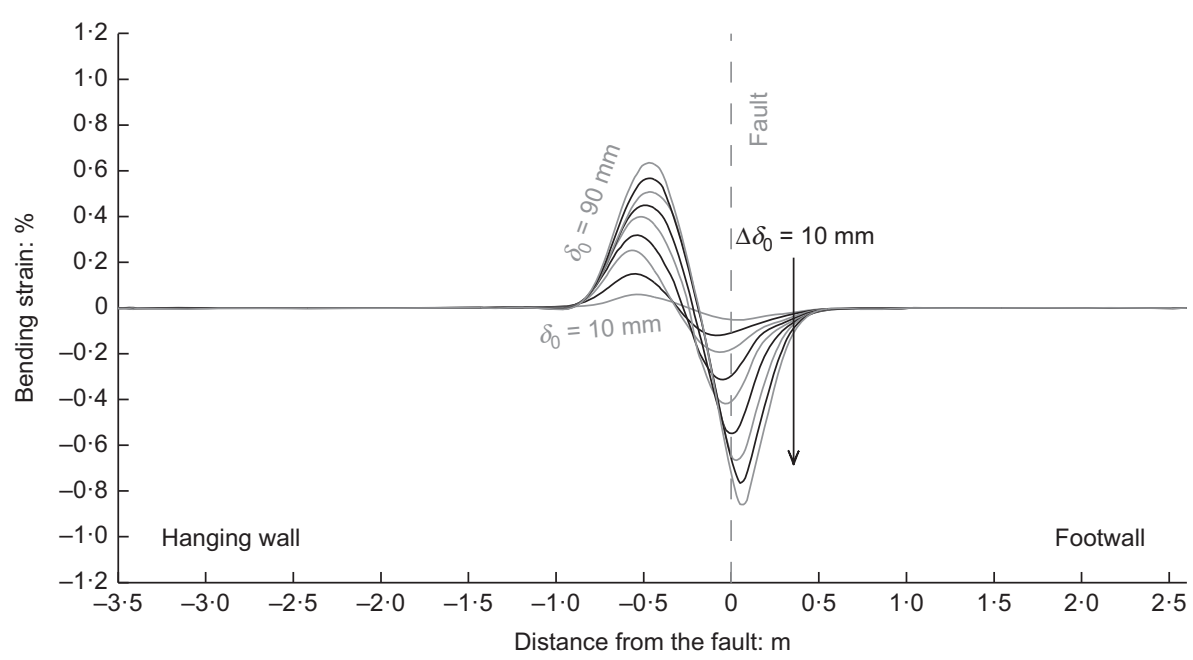

(b)

Fig. 13. Bending strain distribution of (a) 2 inch and (b) 1 inch pipes at various offsets as functions of distance from the fault

of axial strain along the length of the pipe can also be found in the small-diameter pipe tests.

\section{Comparison with existing analysis methods}

The peak pipe responses to normal ground faults are plotted in Fig. 15, where both the axial and bending strains are proportional to the normalised fault offset. The markers and error bars in the axial strain plot show the variations between measured (see Fig. 14(a)) and interpreted responses (see Fig. 14(b)) respectively. Consistent results have been obtained for the 4 inch pipe tests with different types of instrumentation. The difference in measured peak strains for the two sensing systems could have partially resulted from the coating treatment, where a multi-layer waterproof protection system was applied to the strain-gauged pipe, while the fibre optic sensors were only covered with a silicone sealant. As expected, design should be dominated by considerations of the flexural response of the pipe in the hogging zone, where larger peak bending strains occur. Axial and bending strains increase with pipe diameter given a fixed value of Young's modulus for the pipe. Larger diameter indicates more surface area where friction is mobilised and large axial forces and axial strains develop along the pipe. Bending strain, $\varepsilon_{\mathrm{b}}=\kappa D / 2$, is simply a product of curvature $\kappa$ and the distance from the neutral axis $D / 2$. The peak axial strains are less than $0 \cdot 15 \%$, which are one order of magnitude smaller than those measured for bending strains at about 1\%. A slight deviation from linear behaviour can be observed where the flexural response starts to soften at the final stage of the faulting action.

Based on the bending strain calculation, the variations of $i_{\text {pipe }}$ values with fault offset are presented in Fig. 16, where an almost independent relationship can be seen, especially for the more flexible, smaller diameter pipes. The influence of pipe stiffness on the length scale of bending is indicated in Fig. 16, as higher flexibility pipes have smaller $i_{\text {pipe }}$ values, indicative of a shorter length scale for flexure (Saiyar, 2011; $\mathrm{Ni}, 2016$ ). Different governing mechanisms on both sides of the fault contribute to the difference of $i_{\text {pipe }}$ values in the hogging and sagging zones. An abrupt change in length scale (i.e. a smaller $i_{\text {pipe }}$ value) corresponds to a zone of high stress concentration within the pipe that yields larger flexural strains.

The current design approaches often treat the pipe as a beam or shell structure (Xie et al., 2011, 2013), where it is surrounded by a series of discrete, independent, bilinear spring elements. To provide conservative calculations, the spread of displacement discontinuity with the fault propagation from the bedrock up to the ground surface is generally ignored; instead, an abrupt step-like fault (the motion generated at the bedrock) is assumed at the pipe burial depth. For the normal fault-pipeline interaction problem, the horizontal springs can be neglected as they provide no 


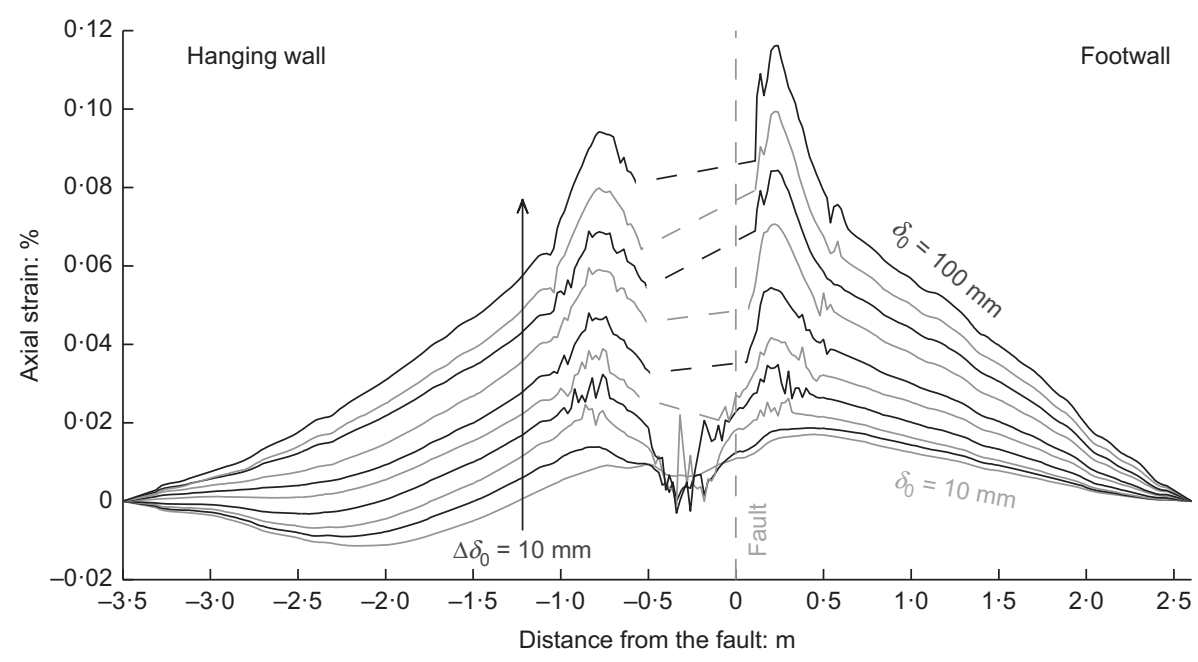

(a)

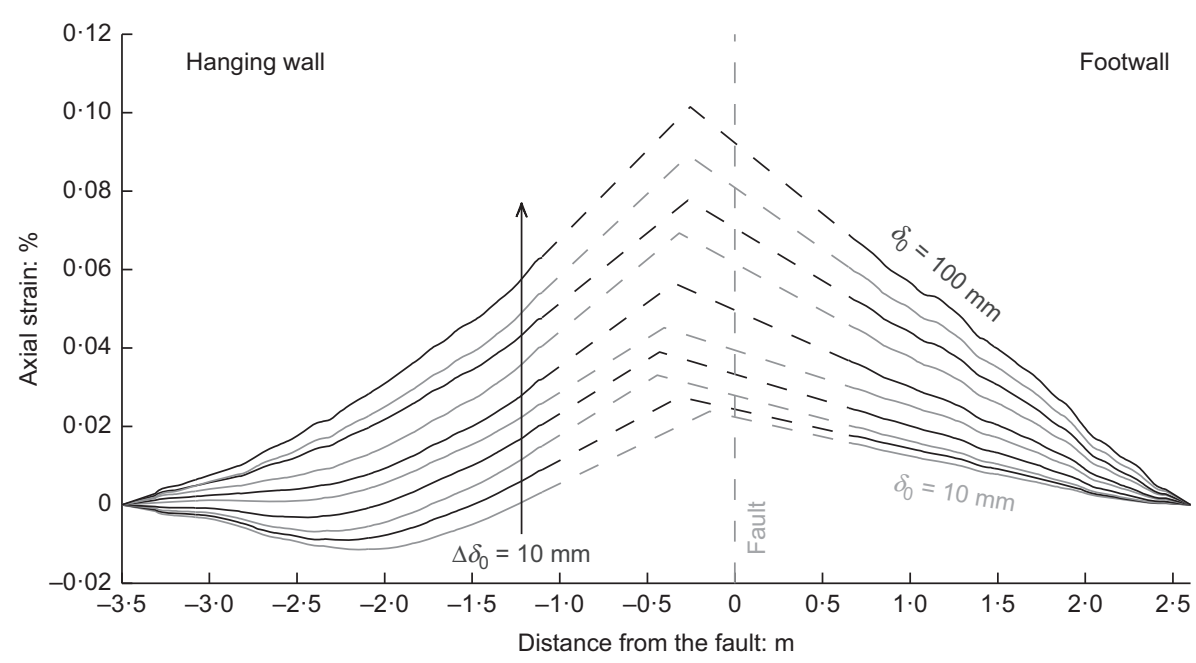

(b)

Fig. 14. Axial strain distribution of 2 inch pipe at various offsets as functions of distance from the fault: (a) measured response; (b) interpreted response at bad correlation zone

contribution to soil-pipe resistance. Based on the American Lifeline Alliance (ALA, 2001) guidelines, the properties of soil springs for the test pipes are calculated as shown in Table 2. Two existing analysis approaches are considered in this investigation, namely the Winkler analysis (ALA, 2001) and the three-beam model of Karamitros et al. (2011), to check their ability to estimate peak bending response for flexible pipes. In addition, the distributed fault displacements measured in the free-field test provide the opportunity to distinguish properly the effects of the imposed displacement pattern from the effects of spring stiffness.

Figure 17 presents the variation of experimentally measured peak bending strains with normalised fault offset, $\delta_{0} / D$, for the test pipes with different diameters using filled square markers. Given that the peak strains observed in the 4 inch pipe instrumented with strain gauges were equivalent to those obtained using fibre optic sensing (see Fig. 15), only fibre optic data are incorporated in Fig. 17 for the sake of clarity. These experimental observations are then compared with the results from the three-beam method and a variety of different Winkler analyses investigating calculation procedures using the existing default analysis for rigid pipes (e.g. empirical soil springs and a knife-edge displacement) and modifications to either impose the free-field deformation profile or reduce the stiffness of the empirical soil springs.
Winkler analyses are presented in Fig. 17 using light grey lines to denote the use of the distributed fault displacement and spring icons with fewer coils to represent the use of a reduction factor of $1 / 3$ on spring stiffness.

This comparison clearly illustrates the shortcomings of the existing beam-on-spring procedure and three-beam method as the pipe becomes more and more flexible (i.e. as diameter decreases), because these calculations are very dependent on empirical soil springs that were empirically calibrated for high-stiffness (e.g. steel) pipelines. It is valuable, therefore, to explore alternatives for analysis of flexible pipes subjected to differential ground motion. Saiyar et al. (2016) and Xie et al. (2013) both used a reduction factor for soil springs to improve the performance of current analysis techniques. Therefore, a similar strategy was adopted in the current paper, incorporating a scale factor of $1 / 3$ with the peak force that develops in a vertical bearing spring (O'Rourke et al., 2015). Marked improvements in the calculated values of peak bending strain can be seen. The beneficial effects of using a distributed fault profile alone can also be observed as given, but it is evident that this strategy is less effective than that using reduced spring stiffness. Incorporating both the modified soil springs and the distributed fault displacement in the calculations does not provide significant improvement compared to the analyses using modified soil springs alone. 


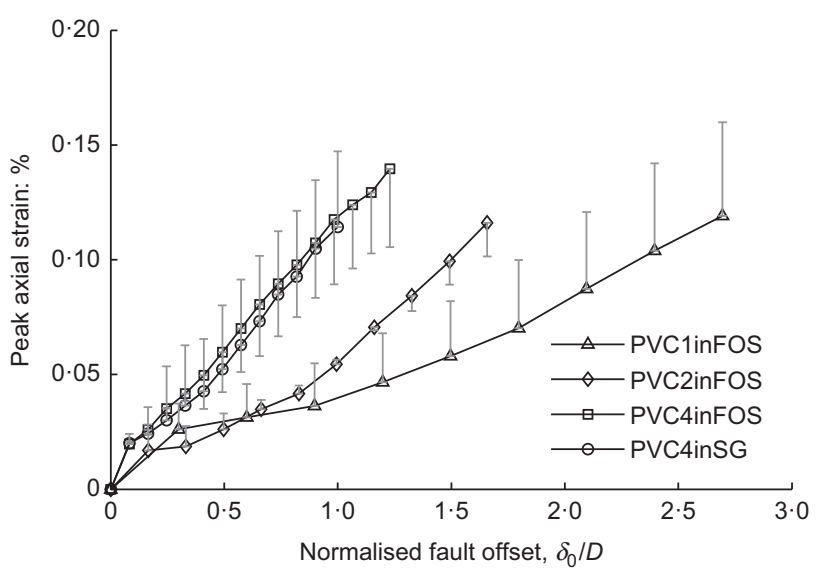

(a)

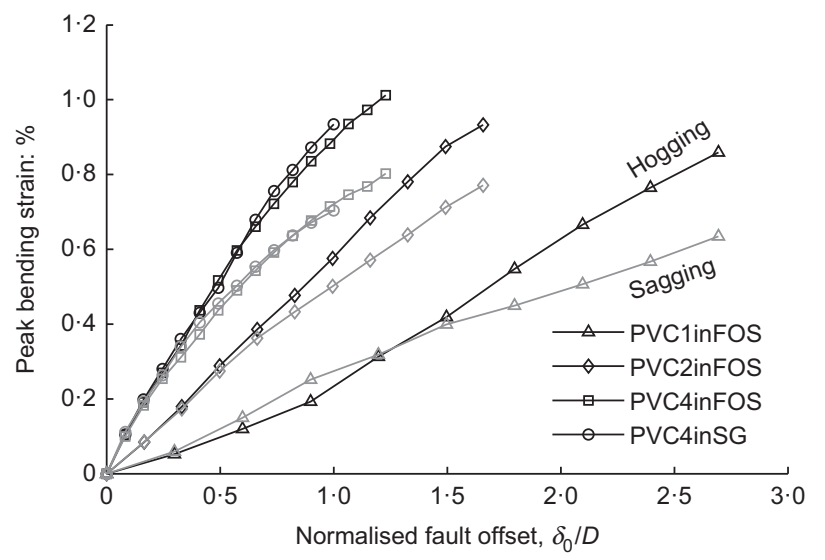

(b)

Fig. 15. Peak pipe responses: (a) axial and (b) bending strain as functions of various normalised fault offsets

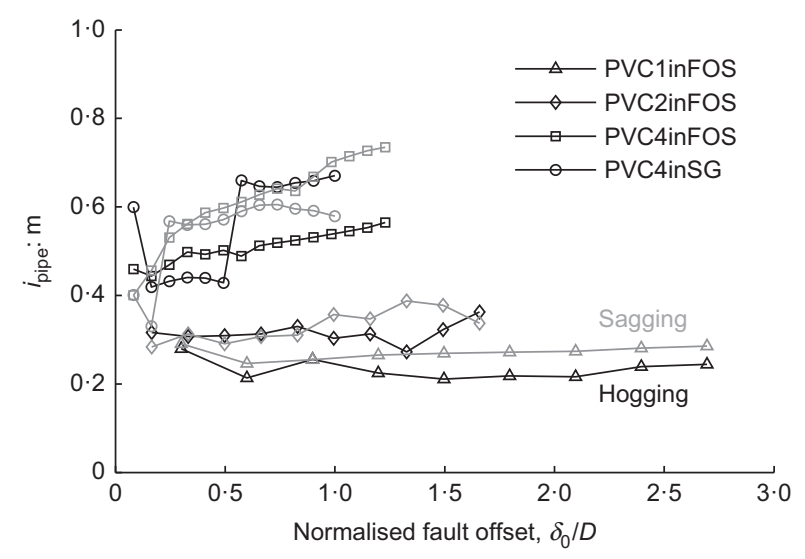

Fig. 16. Variation of $i_{\text {pipe }}$ values with fault offset showing the impact of pipe stiffness

\section{CONCLUSIONS}

Four full-scale buried pipe tests were carried out in the newly assembled split-box apparatus at Queen's University to investigate the performance of pipelines buried in dry sand crossing normal ground faults at $90^{\circ}$. PVC pipe was selected due to the lack of prototype-scale experimental evidence for flexible pipes under differential ground motion in the vertical plane. This choice was made to test a pipe with a large range of linear elasticity, so the influence of non-linear and time-dependent constitutive behaviour for the pipe material (features of many other polymer materials) could be eliminated as an influencing factor.

Pipe ends were left free to simulate a $6 \mathrm{~m}$ long pipe segment between two adjacent gasketed joints in a continuous piping system. At the joints, the pipe ends slip, so the axial strains develop along much of the pipe length. Release of axial force at the pipe ends induces higher levels of flexural response (greater magnitudes of maximum curvature), and so more conservative values of flexural strain compared to those that result if the ends are restrained (restraint leads to axial tension that suppresses flexure).

The axial and bending strains were calculated from pairs of longitudinal strain values along the crown and invert (the extreme fibres) of the pipe during fault rupture propagation. Comparison of the results obtained from 4 inch pipes using different instrumentation provides confidence that the fibre optic sensing technique provides effective measurements of distributed strain along the pipeline at a spacing of $20 \mathrm{~mm}$. This measurement resolution is not available when using conventional strain gauges, unless a very large number of gauges are installed (not feasible given the time and expense associated with installation of many conventional gauges). However, the nylon-coated fibre can only provide strain measurements of up to $1 \%$ before it breaks. Given the satisfactory performance of nylon-coated fibre, this instrumentation was also adopted for two small-diameter (1 inch and 2 inch) pipe tests.

Under normal faulting, the pipeline experiences downwards and upheaval motion above the stationary and translating sections of the floor, respectively, with these motions governed by bearing and uplift capacity of the soil under and over the pipe, respectively. The unsymmetric behaviour observed in distributions of both axial and bending strains, is attributed to the different soil-pipe interaction mechanisms on either side of the fault. Larger soil reactions on the footwall side lead to a zone of high stress concentration, resulting in the peak axial and bending strains occurring over this footwall side. A second local peak value of axial strains occurs on the hanging wall side and the strain distribution between two peaks dips down proximal to the point of contraflexure in the bending response. The normal fault-pipeline interaction problem is dominated by the flexural (bending) strains, which are one order of magnitude greater than the axial strains observed at the pipe midsurface. There is an approximately linear relationship between the strains that develop and the fault offset. A slight softening behaviour was observed in the peak bending strains. For a given pipe material, diameter plays an important role in altering pipe responses, since axial strain is directly related to the pipe's external surface area, and bending strain is proportional to the distance of the extreme fibre from the neutral axis (half the diameter).

A comparison was made between the peak bending strains measured in the experiments and calculations using existing procedures (i.e. the Winkler-based numerical analysis and the three-beam model). These existing approaches all use empirical spring elements to approximate the surrounding soil, so they all feature the same limitations related to the values of spring stiffness. In design guidelines, soil springs are defined based on the data of model-scale laboratory tests on high-stiffness (steel) pipelines or other similar structures, such as plate anchors or piles. The performance of the existing modelling techniques in analysis of flexible pipes may be affected by the use of springs back-calculated from tests on stiff steel pipelines, and the current study demonstrated that those analysis procedures are very conservative, especially for flexible pipes (with small diameter). A modified technique involving use of spring stiffness that is $1 / 3$ of the steel pipe values has been tested and found to provide much better 


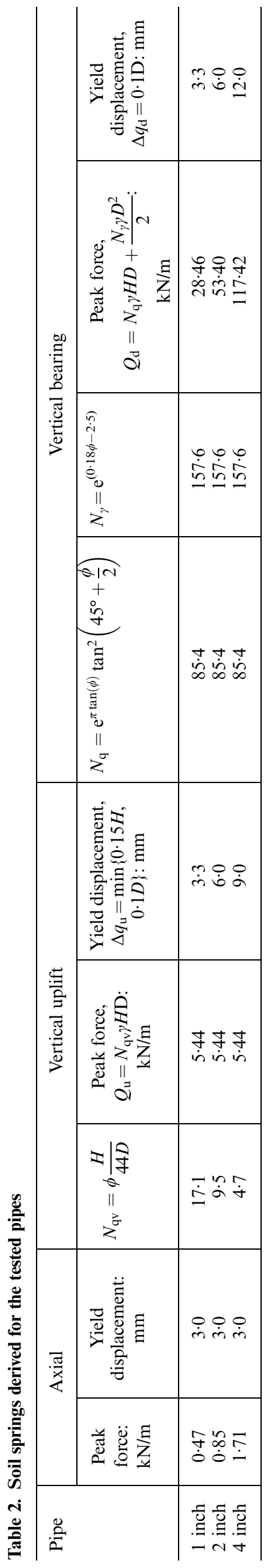

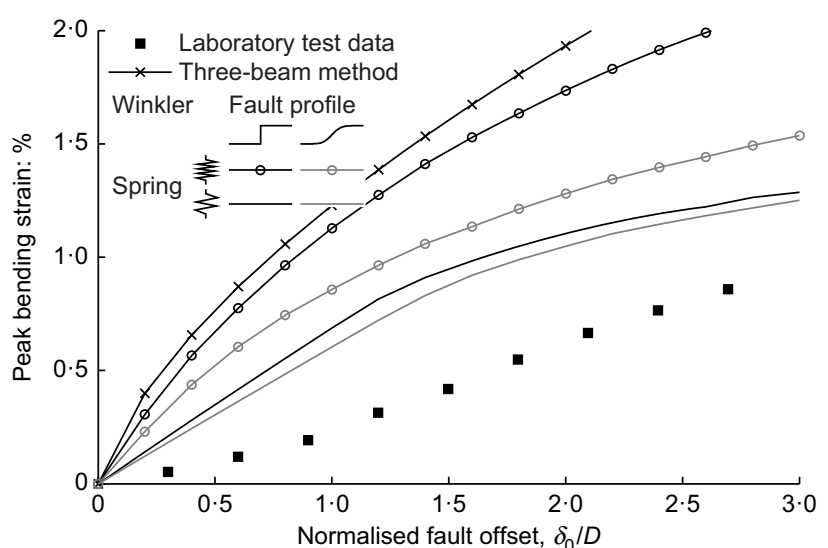

(a)

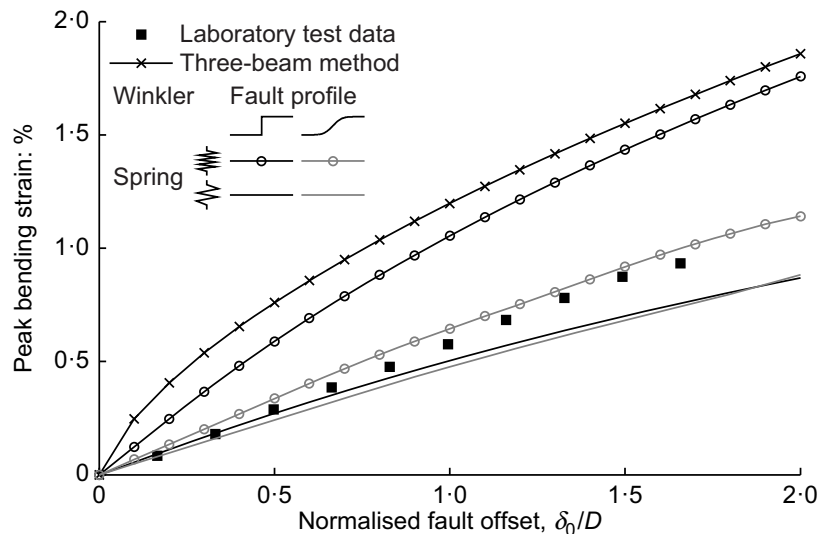

(b)

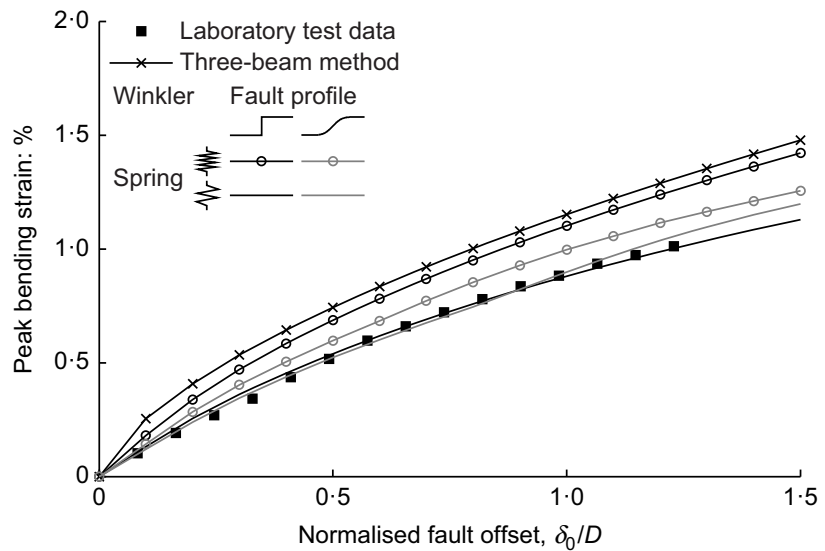

(c)

Fig. 17. Comparison of measured and calculated maximum bending strains: (a) 1 inch pipe; (b) 2 inch pipe; (c) 4 inch pipe. Winkler analyses are presented investigating the use of the distributed fault displacement (light grey) as well as a reduction value of 1/3 on spring stiffness (spring icon with fewer coils)

results. The measurements of free-field soil response provide the opportunity to check whether imposing distributed fault displacements can improve the analyses, but it was found to be less effective than modifying spring stiffness.

\section{ACKNOWLEDGEMENTS}

Financial contributions from the Natural Sciences and Engineering Research Council of Canada (NSERC) through a Strategic Research Grant, a Major Resources Support Grant and Discovery Grants to Drs Ian D. Moore and W. Andy Take made this study possible; financial support from a student scholarship to the first author from an 
NSERC CREATE grant associated with the STEWARD programme at Queen's and Dalhousie Universities is also gratefully acknowledged.

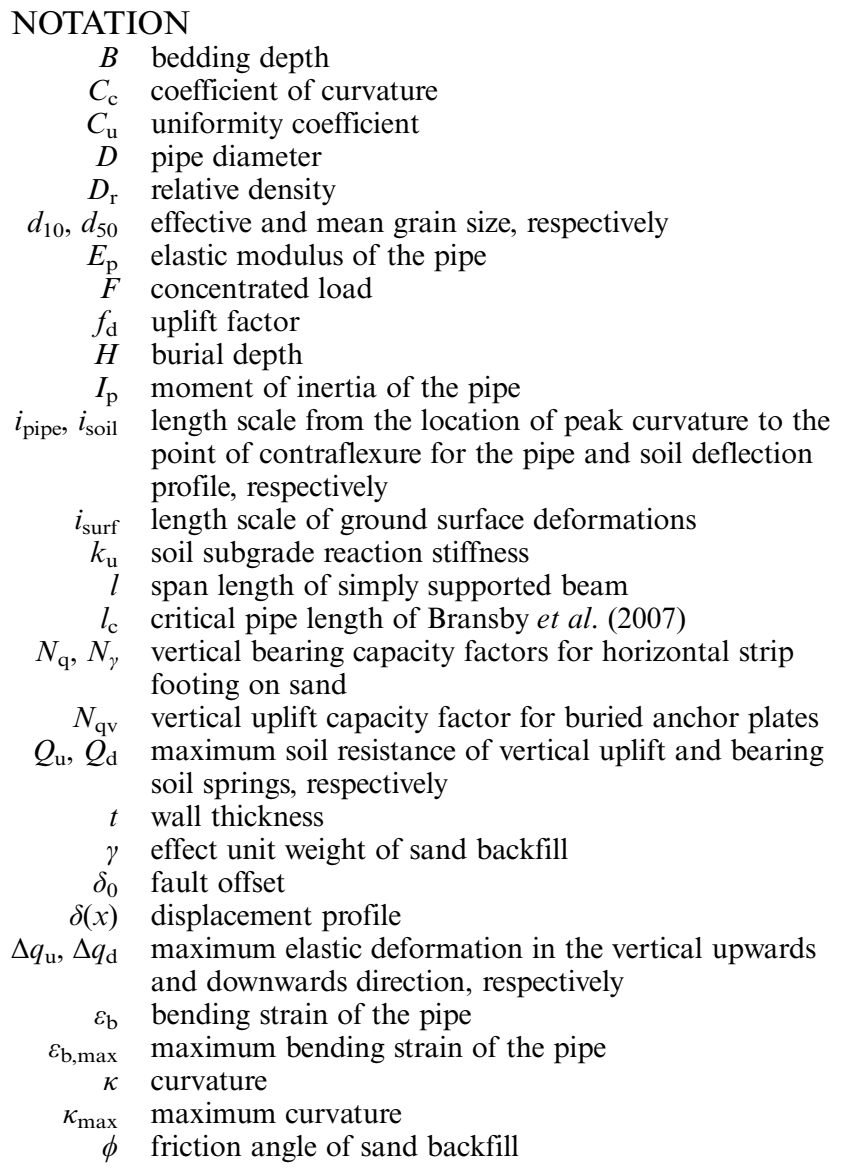

\section{REFERENCES}

Abdoun, T. H., Ha, D., O'Rourke, M. J., Symans, M. D., O'Rourke, T. D., Palmer, M. C. \& Stewart, H. E. (2009). Factors influencing the behavior of buried pipelines subjected to earthquake faulting. Soil Dynamics Earthquake Engng 29, No. 3, 415-427.

Agrawal, G. P. (2006). Nonlinear fiber optics, 4th edn. Rochester, NY, USA: Academic Press.

ALA (American Lifeline Alliance) (2001). Guidelines for the design of buried steel pipe. Washington, DC, USA: American Lifeline Alliance (ALA), a public-private partnership between American Society of Civil Engineers (ASCE) and Federal Emergency Management Agency (FEMA).

ASTM (2015). D1785-15: Standard Specification for Poly(Vinyl Chloride) (PVC) Plastic Pipe, Schedules 40, 80, and 120. West Conshohocken, PA, USA: ASTM International.

AWWA (American Water Works Association) (2007). C900-07: Polyvinyl Chloride (PVC) Pressure Pipe and Fabricated Fittings, 4 In. Through 12 In. (100 mm Through $300 \mathrm{~mm}$ ), for Water Transmission and Distribution. Denver, CO, USA: American Water Works Association.

Bransby, M. F., EI Nahas, A., Turner, E. \& Davies, M. C. R. (2007). The interaction of reverse faults with flexible continuous pipelines. Int. J. Phys. Modelling Geotech. 7, No. 3, 25-40.

Burnett, A. J. (2015). Investigation of full scale horizontal pipe-soil interaction and large strain behaviour of sand. Master thesis, Queen's University, Kingston, ON, Canada.

Cheuk, C. Y., White, D. J. \& Bolton, M. D. (2008). Uplift mechanisms of pipes buried in sand. J. Geotech. Geoenviron. Engng 134, No. 2, 154-163.

Cholewa, J. A., Brachman, R. W. I. \& Moore, I. D. (2009). Response of a polyvinyl chloride water pipe when transverse to an underlying pipe replaced by pipe bursting. Can. Geotech. J. 46, No. 11, 1258-1266.

Choo, Y. W., Abdoun, T. H., O’Rourke, M. J. \& Ha, D. (2007). Remediation for buried pipeline systems under permanent ground deformation. Soil Dynamics Earthquake Engng 27, No. 12, 1043-1055.

Erami, M. H., Miyajima, M., Kaneko, S., Toshima, T. \& Kishi, S. (2015). Pipe-soil interaction for segmented buried pipelines subjected to dip faults. Earthquake Engng Struct. Dynamics 44, No. 3, 403-417.

Gabriel, L. (ed.) (2011). Design methodology. In Corrugated polyethylene pipe design manual \& installation guide, chapter 5. Irving, TX, USA: Plastic Pipe Institute. See http:// rothengaran.com/uploads/chapter-5_design_method.pdf (accessed 28/04/2017).

Groves, J. \& Wijewickreme, D. (2013). Field monitoring of buried polyethylene natural gas pipelines subjected to ground movement. GéoMontréal 2013, proceedings of the 66th Canadian geotechnical conference and the 11th joint CGS/IAH-CNC groundwater conference, Montreal, QC, Canada.

G'Sell, C. \& Jonas, J. J. (1981). Yield and transient effects during the plastic deformation of solid polymers. J. Mater. Sci. 16, No. 7, 1956-1974.

Ha, D., Abdoun, T. H., O'Rourke, M. J., Symans, M. D., O'Rourke, T. D., Palmer, M. C. \& Stewart, H. E. (2008a). Buried high-density polyethylene pipelines subjected to normal and strike-slip faulting - a centrifuge investigation. Can. Geotech. $J$. 45, No. 12, 1733-1742.

Ha, D., Abdoun, T. H., O’Rourke, M. J., Symans, M. D., O’Rourke, T. D., Palmer, M. C. \& Stewart, H. E. (2008b). Centrifuge modeling of earthquake effects on buried high-density polyethylene (HDPE) pipelines crossing fault zones. J. Geotech. Geoenviron. Engng 134, No. 10, 1501-1515.

Ha, D., Abdoun, T. H., O’Rourke, M. J., Symans, M. D., O'Rourke, T. D., Palmer, M. C. \& Stewart, H. E. (2010). Earthquake faulting effects on buried pipelines - case history and centrifuge study. J. Earthquake Engng 14, No. 5, 646-669.

Jung, J. K., O'Rourke, T. D. \& Olson, N. A. (2013a). Lateral soil-pipe interaction in dry and partially saturated sand. J. Geotech. Geoenviron. Engng 139, No. 12, 2028-2036.

Jung, J. K., O'Rourke, T. D. \& Olson, N. A. (2013b). Uplift soil-pipe interaction in granular soil. Can. Geotech. J. 50, No. 7, 744-753.

Karamitros, D. K., Bouckovalas, G. D. \& Kouretzis, G. P. (2007). Stress analysis of buried steel pipelines at strike-slip fault crossings. Soil Dynamics Earthquake Engng 27, No. 3, 200-211.

Karamitros, D. K., Bouckovalas, G. D., Kouretzis, G. P. \& Gkesouli, V. (2011). An analytical method for strength verification of buried steel pipelines at normal fault crossings. Soil Dynamics Earthquake Engng 31, No. 11, 1452-1464.

Kennedy, R. P., Chow, A. W. \& Williamson, R. A. (1977). Fault movement effects on buried oil pipeline. J. Transpn Engng 103, No. 5, 617-633.

Klar, A. \& Marshall, A. M. (2008). Shell versus beam representation of pipes in the evaluation of tunneling effects on pipelines. Tunnelling Underground Space Technol. 23, No. 4, 431-437.

Klar, A., Vorster, T. E. B., Soga, K. \& Mair, R. J. (2005). Soil-pipe interaction due to tunnelling: comparison between Winkler and elastic continuum solutions. Géotechnique 55, No. 6, 461-466, http://dx.doi.org/10.1680/geot.2005.55.6.461.

Klar, A., Marshall, A. M., Soga, K. \& Mair, R. J. (2008). Tunneling effects on jointed pipelines. Can. Geotech. J. 45, No. 1, 131-139.

Kouretzis, G. P., Karamitros, D. K. \& Sloan, S. W. (2015). Analysis of buried pipelines subjected to ground surface settlement and heave. Can. Geotech. J. 52, No. 8, 1058-1071.

Li, H., Xue, N., Li, X. \& Li, Z. (2012a). An in-situ experimental study on buried pipelines with internal pressure subject to a simulated reverse-slip fault movement. Proceedings of the 15th world conference on earthquake engineering (15WCEE), Lisbon, Portugal.

Li, X., Wang, B. \& Zhou, J. (2012b). Analytical method of submarine buried steel pipelines under strike-slip faults. Proceedings of the 15th world conference on earthquake engineering (15WCEE), Lisbon, Portugal.

Luna Technologies (2011). Optical backscatter reflectometer model 4600 user guide: version 3. Blacksburg, VA, USA: Luna Technologies. 
Mair, R. J., Taylor, R. N. \& Bracegirdle, A. (1993). Subsurface settlement profiles above tunnels in clays. Géotechnique 43, No. 2, 315-320, http://dx.doi.org/10.1680/geot.1993.43.2.315.

Marshall, A. M., Klar, A. \& Mair, R. J. (2010). Tunneling beneath buried pipes: view of soil strain and its effect on pipeline behavior. J. Geotech. Geoenviron. Engng 136, No. 12, $1664-1672$.

Marshall, A. M., Farrell, R., Klar, A. \& Mair, R. (2012). Tunnels in sands: the effect of size, depth and volume loss on greenfield displacements. Géotechnique 62, No. 5, 385-399, http://dx.doi. org/10.1680/geot.10.P.047.

Moore, I. (2001). Buried pipes and culverts. In Geotechnical and geoenvironmental engineering handbook (ed. R. K. Rowe), pp. 541-567. Norwell, MA, USA: Springer US, Kluwer Academic Publishers.

Newmark, N. M. \& Hall, W. J. (1975). Pipeline design to resist large fault displacement. Proceedings of U.S. national conference on earthquake engineering, Ann Arbor, MI, USA, pp. 416-425.

$\mathrm{Ni}, \mathrm{P}$. (2016). Nonlinear soil-structure interaction for buried pressure pipes under differential ground motion. PhD thesis, Queen's University, Kingston, ON, Canada.

O'Rourke, M. J. \& Liu, X. (2012). Seismic design of buried and offshore pipelines, Monograph MCEER-12-MN04. Buffalo, NY, USA: Multidisciplinary Center for Earthquake Engineering Research, University at Buffalo.

O'Rourke, T. D., Jezerski, J. M., Olson, N. A., Bonneau, A. L., Palmer, M. C., Stewart, H. E., O'Rourke, M. J. \& Abdoun, T. (2008). Geotechnics of pipeline system response to earthquakes. In Geotechnical earthquake engineering and soil dynamics IV (eds D. Zeng, M. T. Manzari and D. R. Hiltunen), Geotechnical Special Publication no. 181. Reston, VA, USA: American Society of Civil Engineers (CD-ROM).

O'Rourke, T. D., Jung, J. K. \& Argyrou, C. (2015). Underground infrastructure response to earthquake-induced ground deformation. Proceedings of the 6th international conference on earthquake geotechnical engineering, Christchurch, New Zealand, p. 20.

Pixa, R., Le Dû, V. \& Wippler, C. (1988). Dilatometric study of deformation induced volume increase and recovery in rigid PVC. Colloid Polymer Sci. 266, No. 10, 913-920.

Poon, E. (2015). Jointed pipelines response in moving ground. MASc thesis, Department of Civil Engineering, Queen's University, Kingston, ON, Canada.

Povolo, F., Schwartz, G. \& Hermida, E. B. (1996). Stress relaxation of PVC below the yield point. J. Polymer Sci. Part B Polymer Physics 34, No. 7, 1257-1267.

Rojhani, M., Moradi, M., Galandarzadeh, A. \& Takada, S. (2012). Centrifuge modeling of buried continuous pipelines subjected to reverse faulting. Can. Geotech. J. 49, No. 6, 659-670.

Saiyar, M. (2011). Behaviour of buried pipelines subject to normal faulting. $\mathrm{PhD}$ thesis, Queen's University, Kingston, $\mathrm{ON}$, Canada.

Saiyar, M., Take, W. A. \& Moore, I. D. (2011). Validation of boundary PIV measurements of soil-pipe interaction. Int. J. Phys. Modelling Geotech. 11, No. 1, 23-32.

Saiyar, M., Ni, P., Take, W. A. \& Moore, I. D. (2016). Response of pipelines of differing flexural stiffness to normal faulting. Géotechnique 66, No. 4, 275-286, http://dx.doi.org/10.1680/ jgeot.14.P.175.

Sim, W. W., Towhata, I. \& Yamada, S. (2012a). One-g shaking-table experiments on buried pipelines crossing a strike-slip fault. Géotechnique 62, No. 12, 1067-1079, http://dx.doi.org/10.1680/ geot.10.P.142.
Sim, W. W., Towhata, I., Yamada, S. \& Moinet, G. J. M. (2012b). Shaking table tests modelling small diameter pipes crossing a vertical fault. Soil Dynamics Earthquake Engng 35, $59-71$.

Simpson, B., Hoult, N. A. \& Moore, I. D. (2015). Distributed sensing of circumferential strain using fiber optics during full-scale buried pipe experiments. J. Pipeline Systems Engng Practice, 6, No. 4, 04015002.

Stanier, S. A., Blaber, J., Take, W. A. \& White, D. J. (2015). Improved image-based deformation measurement for geotechnical applications. Can. Geotech. J. 53, No. 5, 727-739.

Takada, S., Hassani, N. \& Fukuda, K. (2001). A new proposal for simplified design of buried steel pipes crossing active faults. Earthquake Engng Struct. Dynamics 30, No. 8, 1243-1257.

Take, W. A. (2015). Thirty-sixth Canadian geotechnical colloquium: advances in visualization of geotechnical processes through digital image correlation. Can. Geotech. J. 52, No. 9, 1199-1220.

Tognon, A. R., Rowe, R. K. \& Brachman, R. W. I. (1999). Evaluation of side wall friction for a buried pipe testing facility. Geotextiles and Geomembranes 17, No. 4, 193-212.

Trifonov, O. V. \& Cherniy, V. P. (2010). A semi-analytical approach to a nonlinear stress strain analysis of buried steel pipelines crossing active faults. Soil Dynamics Earthquake Engng 30, No. $11,1298-1308$.

Trifonov, O. V. \& Cherniy, V. P. (2012). Elastoplastic stress-strain analysis of buried steel pipelines subjected to fault displacements with account for service loads. Soil Dynamics Earthquake Engng 33, No. 1, 54-62.

Vorster, T. E. B., Klar, A., Soga, K. \& Mair, R. J. (2005). Estimating the effects of tunneling on existing pipelines. J. Geotech. Geoenviron. Engng 131, No. 11, 1399-1410.

Wang, B., Li, X. \& Zhou, J. (2011). Strain analysis of buried steel pipelines across strike-slip faults. J. Central South Univ. Technol. 18, No. 5, 1654-1661.

Wang, L. R. L. \& Yeh, Y. H. (1985). A refined seismic analysis and design of buried pipeline for fault movement. Earthquake Engng Struct. Dynamics 13, No. 1, 75-96.

Wham, B. P., Argyrou, C., O'Rourke, T. D., Stewart, H. E. \& Bond, T. K. (2016). PVCO pipeline performance under large ground deformation. J. Pressure Vessel Technol. 139, No. 1, 011702

White, D. J., Barefoot, A. J. \& Bolton, M. D. (2001). Centrifuge modelling of upheaval buckling in sand. Int. J. Phys. Modelling Geotech. 1, No. 2, 19-28.

White, D. J., Take, W. A. \& Bolton, M. D. (2003). Soil deformation measurement using particle image velocimetry (PIV) and photogrammetry. Géotechnique 53, No. 7, 619-631, http://dx. doi.org/10.1680/geot.53.7.619.37383.

White, D. J., Cheuk, C. Y. \& Bolton, M. D. (2008). The uplift resistance of pipes and plate anchors buried in sand. Géotechnique 58, No. 10, 771-779, http://dx.doi.org/10.1680/ geot.2008.3692.

Xie, X. J., Symans, M. D., O'Rourke, M. J., Abdoun, T. H., O'Rourke, T. D., Palmer, M. C. \& Stewart, H. E. (2011). Numerical modeling of buried HDPE pipelines subjected to strike-slip faulting. J. Earthquake Engng 15, No. 8, 1273-1296.

Xie, X., Symans, M. D., O'Rourke, M. J., Abdoun, T. H., O'Rourke, T. D., Palmer, M. C. \& Stewart, H. E. (2013). Numerical modeling of buried HDPE pipelines subjected to normal faulting: a case study. Earthquake Spectra 29, No. 2, 609-632.

Yoshizaki, K., O’Rourke, T. D. \& Hamada, M. (2003). Large scale experiments of buried steel pipelines with elbows subjected to permanent ground deformation. Struct. Engng/Earthquake Engng 20, No. 1, 1s-11s. 